Sharif University of Technology
Scientia Iranica
Transactions E: Industrial Engineering
hCIttp://scientiairanica.sharif.edu
IRAN ICA

\title{
Complex q-rung orthopair fuzzy variation coefficient similarity measures and their approach to medical diagnosis and pattern recognition
}

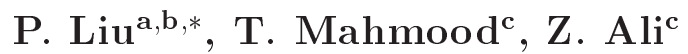 \\ a. School of Management Science and Engineering, Shandong University of Finance and Economics, Jinan 250015, Shandong \\ Province, China. \\ b. School of Economics and Management, Civil Aviation University of China, Tianjin, 300300, China. \\ c. Department of Mathematics \& Statistics, International Islamic University, Islamabad, Pakistan.
}

Received 30 December 2019; received in revised form 3 March 2020; accepted 1 June 2020

\begin{abstract}
KEYWORDS
Complex q-rung orthopair fuzzy sets; Interval-valued complex q-rung orthopair fuzzy sets; Jaccard similarity measures;

Hybrid vector similarity measures; Variation coefficient similarity measures.
\end{abstract}

\begin{abstract}
The q-Rung Orthopair Fuzzy Set (q-ROFS) as a generalization of Fuzzy Set (FS) is characterized by membership and non-membership. The sum of their q-powers is restricted to $[0,1]$. In this study, a new Complex q-ROFS (Cq-ROFS) is proposed by combining q-ROFS with Complex FS (CFS). Cq-ROFS is a better way to process uncertain and imprecise information in decision-making, which is characterized by complex-valued membership and complex-valued non-membership. First, some fundamental operational laws, score function and accuracy function, and a comparison method are proposed. Further, because the Vector Similarity Measures (VSMs) play a key role in statistics, physics, and engineering, some VSMs including Jaccard Similarity Measures (JSMs), Dice Similarity Measures (DSMs), and Cosine Similarity Measures (CSMs) for Cq-ROFSs and Interval-Valued Cq-ROFS (IVCq-ROFS) are investigated. Moreover, Hybrid VSMs (HVSMs) called Variation Co-efficient Similarity Measures (VCSMs) for Cq-ROFSs and IVCq-ROFSs are also proposed and their properties are discussed. Finally, in order to demonstrate the feasibility of the investigated HVSMs, the existing similarity measures about Complex Pythagorean Fuzzy Sets (CPFSs) and Complex Intuitionistic Fuzzy Sets (CIFSs) are compared with the proposed methods by numerical examples of medical diagnosis and pattern recognition.

(C) 2022 Sharif University of Technology. All rights reserved.
\end{abstract}

\section{Introduction}

Intuitionistic Fuzzy Set (IFS), initiated by Atanassove [1], is an extension of Fuzzy Set (FS) [2] and is characterized by membership and non-membership grades, the sum of which is restricted to $[0,1]$. IFS is an

*. Corresponding author. Tel.: +86-531-82222188 E-mail addresses: peide.liu@gmail.com (P. Liu); tahirbakhat@iiu.edu.pk (T. Mahmood); zeeshan.msma434@iiu.edu.pk (Z.Ali)

doi: $10.24200 /$ sci.2020.55133.4089 important tool to deal with awkward and complicated information in real decision problems and now, it is receiving greater attention in the domain of distance measures [3-5] and entropy measures for IFSs [6-8]. De et al. [9] introduced the application of IFSs in medical diagnosis. Szmidt and Kacprzyk $[10,11]$ utilized IFS to process the medical diagnosis problems. Wei [12] established geometric aggregation operators, and $\mathrm{Xu}$ [13] established power aggregation operators based on IFS. Liu [14] developed Hamacher aggregation operators based on interval-valued IFS. Further, Alkouri and Salleh [15] initiated the idea of Complex IFSs (CIFSs) as a generalization of Complex FS (CFS) [16]. The 
CIFS is characterized by complex-valued membership and complex-valued non-membership functions, whose range is not limited to $[0,1]$ but extended to unit disc in the complex plane. Moreover, Kumar and Bajaj [17] combined the notions of CIFS and soft sets [18] and proposed complex intuitionistic fuzzy soft sets. At the same time, complex intuitionistic fuzzy relation was developed by Alkouri and Salleh [19]. Recently, Rani and Garg [20,21] gave different ideas of distance measures and power aggregation operators based on CIFS. Garg and Rani [22,23] developed different ideas of power aggregation operators and robust correlation coefficient for CIFS in Multi-Attribute Group DecisionMaking (MAGDM) problems.

In addition, the idea of Pythagorean Fuzzy Set (PFS), initiated by Yager [24], is characterized by membership and non-membership grades whose constraint is that the sum of the square of membership and square of non-membership grades is limited to $[0,1]$. Yager and Abbasov [25] provided an example to explain the meaning of PFS. When a decision-maker provides $\frac{\sqrt{3}}{2}$ for membership grades and $\frac{1}{2}$ for nonmembership grades, the sum of two values is greater than 1 and hence, they cannot be expressed by an IFS, but described by PFS since:

$$
\left(\frac{\sqrt{3}}{2}\right)^{2}+\left(\frac{1}{2}\right)^{2} \leq 1 .
$$

It is clear that the PFS is more general than IFS in coping with uncertain information. Further, Ullah et al. [26] proposed the notion of Complex PFS (CPFS) as a generalization of CIFS and CFS to describe effectively complicated information in the environment of MAGDM problems. Obviously, CPFS is superior to CIFS, CFS, IFS, and FS in dealing with uncertain and complex information in real-life problems.

However, when a decision-maker provides 0.8 for membership and 0.9 for non-membership grades, the sum of the square of membership grade and square of non-membership grade is greater than 1 . Hence, they cannot be expressed by PFS and IFS, but are expressible by q-Rung Orthopair Fuzzy Set (q-ROFS), which was proposed by Yager [27]. The constraint of qROFS is that the sum of q-power of membership grade and q-power of non-membership grade is limited to $[0,1]$. Obviously, when $q=1, \mathrm{q}$-ROFS is reduced to IFS; when $q=2$, it is reduced to PFS, i.e., q-ROFS is more general than PFS, IFS, and FS, and now it has attracted much attention. Liu et al. [28] proposed a Multiple Attribute Decision-Making (MADM) method under q-ROFS. Liu et al. [29] developed the q-rung orthopair fuzzy heronian mean operators. Wang et al. [30] investigated a number of similarity measures for q-ROFS. Jan et al. [31] proposed the idea of qrung orthopair fuzzy graph. Liu and Liu [32] developed q-rung orthopair fuzzy Bonferroni mean operators for aggregating the q-rung orthopair fuzzy numbers. Liu and Wang [33,34] proposed Archimedean Bonferroni mean operators for q-ROFSs. Peng et al. [35] suggested the exponential operators for q-ROFS. Liu et al. [36] extended the power Maclaurin symmetric mean operators for q-ROFS. Yang and Peng [37] investigated the idea of q-rung orthopair fuzzy partitioned Bonferroni mean operators. Li et al. [38] developed the q-rung orthopair linguistic heronain mean operators with their application in MAGDM. Liu and Liu [39] further developed the power Bonferroni mean operators for linguistic q-rung orthopair fuzzy numbers.

Although the q-ROFS can describe complicated uncertain information effectively by reducing restrictions of membership grade and nonmembership grade, when a decision-maker provides $(0.91) e^{i} 2 \pi(0.77)$ for the complex-valued membership grade and (0.87) $e^{i} 2 \pi(0.72)$ for the complex-valued nonmembership grade, the CIFS, CPFS, and q-ROFS cannot effectively described them. To handle such types of information, the Complex q-ROFS (Cq-ROFS) becomes a powerful tool to deal with uncertain and complicated information in fuzzy set theory. The advantage of Cq-ROFS is that the sum of q-power of real part (also for imaginary part) of complex-valued membership grade and that (also for imaginary part) of complex-valued non-membership grade is limited to $[0,1]$. The Cq-ROFS is an extension of CIFS and CPFS to cope with complexity of real-life problems.

In addition, the distance and similarity measures are two important tools that deal with the fuzzy information, which are extensively used in pattern recognition [40,41], machine learning [42-44], and MAGDM $[45,46]$. The most popular distance measures include hamming, Euclidean, and generalized distance measures, and the hamming and Euclidean distance measures are the special cases of the generalized distance measures. Based on the importance of similarity and distance measures for q-ROFSs, many researchers have developed some reasonable similarity and distance measures for q-ROFSs. Du [47] investigated the Makowski-type similarity measures for q-ROFS. Peng and Dai [48] developed multiparametric similarity measures for q-ROFS. The similarity and distance measures of q-ROFSs are complementary to each other. The similarity measures quantify the closeness degree between q-ROFSs, while the distance measures depict the difference between q-ROFSs.

However, the above-mentioned similarity and distance measures are defined based on general distance metrics and they have a weaker discrimination capability. Therefore, they may lead to counter-intuitive results in some special cases. Some derived measures have complex forms, yet without a specific physical meaning. It is necessary to develop an effective distance measure with a relatively concise expression and a 
clear physical meaning from both the mathematical and practical points of view. In particular, because the Cq-ROFS can better express the complex uncertain information and it is more general than some other fuzzy sets, it is important to propose some novel similarity and distance measures for Cq-ROFS and then, to use them to solve the MADM or MAGDM problems. Thus, the motivation and goals of this paper are shown as follows:

1. We propose the notions of Cq-ROFS and intervalvalued Cq-ROFS (IVCq-ROFS) and discuss their fundamental properties. At the same time, some numerical examples are given to explain their meanings;

2. The Jaccard Similarity Measures (JSMs), Dice Similarity Measures (DSMs), Cosine Similarity Measures (CSMs), and Vector Similarity Measures (VSMs) for Cq-ROFS and IVCq-ROFS are proposed and then, the Hybrid VSMs (HVSMs) are also presented for them;

3. We utilize some numerical examples in pattern recognition and medical diagnosis to examine the superiority and effectiveness of the proposed methods;

4. The comparisons between the proposed and existing measures are also discussed in detail to examine the validity and superiority of the established measures.

In order to achieve these goals, the construct of this manuscript is shown as follows. Section 2 reviews some basic concepts of q-ROFSs, IVq-ROFSs, $\mathrm{Cq}-\mathrm{ROFSs}$, and IVCq-ROFSs as well as their properties. In addition, the JSMs, DSMs, and CSMs are reviewed. Section 3 proposes the JSMs, DSMs, CSMs, and HVSMs for Cq-ROFSs and IVCq-ROFSs. Based on HVSMs for Cq-ROFSs and IVCq-ROFSs, Section 4 gives some numerical examples about pattern recognition and medical diagnosis to show the superiority and dominance of the proposed methods. The comparisons between the proposed methods and some existing methods are also discussed in detail. The conclusion is given in the last section.

\section{Preliminaries}

The notions of q-ROFSs and Cq-ROFSs are briefly reviewed in this section and then, the JSMs, DSMs, and CSMs are also introduced. Further, the Cq-ROFSs, IVCq-ROFSs, and their fundamental properties are proposed. For convenience, $k$ is used to represent the finite universal set in this article.

\subsection{The q-ROFSs and IVq-ROFSs}

This subsection introduces q-ROFSs and Cq-ROFSs and discusses their basic properties. Further, JSM, DSM, and CSM are discussed.

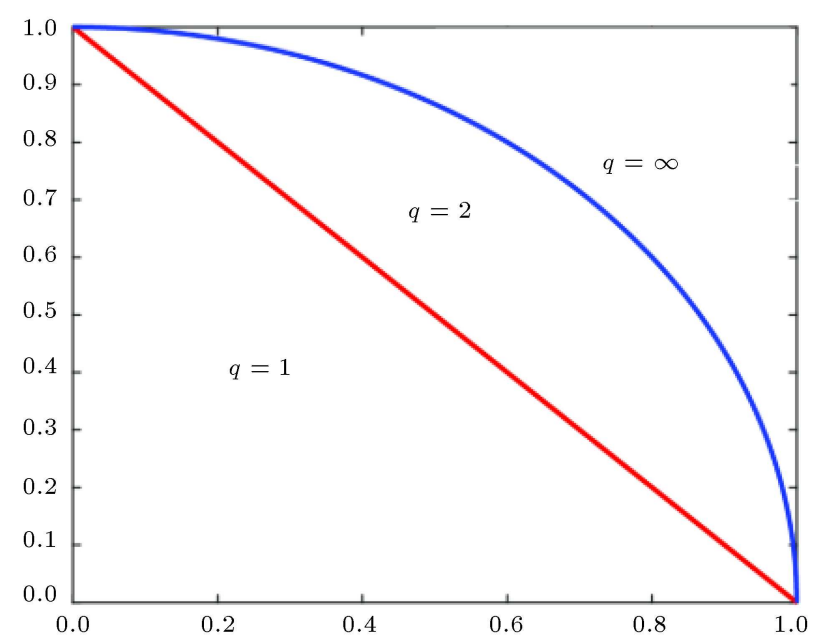

Figure 1. Geometrical interpretation of q-ROFS.

Definition 1 [27]: A q-ROFS is given by:

$$
A=\left\{\left(k, T_{A}^{\prime}(k), F_{A}^{\prime}(k)\right): k \in K\right\},
$$

where $T_{A}^{\prime}, F_{A}^{\prime}: K \rightarrow[0,1]$ represents the membership and non-membership degrees, with a condition that $0 \leq T_{A}^{\prime q}+F_{A}^{\prime q} \leq 1, q \geq 1$. The q-Rung Orthopair Fuzzy Number (q-ROFN) is denoted by $A=\left(T_{A}^{\prime}, F_{A}^{\prime}\right)$. The geometrical interpretation of q-ROFSs is shown in Figure 1.

Definition 2 [27]: Let $A=\left(T_{A}^{\prime}, F_{A}^{\prime}\right)$ be a q-ROFN. Then, the score and accuracy functions of $A$ are given by:

$$
\begin{aligned}
& S(A)=T_{A}^{\prime q}-F_{A}^{\prime q}, S(A) \in[-1,1], \\
& H(A)=T_{A}^{\prime q}+F_{A}^{\prime q}, H(A) \in[0,1] .
\end{aligned}
$$

Definition 3 [27]: Let $A=\left(T_{A}^{\prime}, F_{A}^{\prime}\right)$ and $B=$ $\left(T_{B}^{\prime}, F_{B}^{\prime}\right)$ be two q-ROFNs. Then, the comparison method for q-ROFNs $A$ and $B$ is given as follows:

1. If $S(A) \succ S(B) \Rightarrow A \succ B$;

2. If $S(A)=S(B) \Rightarrow$

1) If $H(A) \succ H(B) \Rightarrow A \succ B$;

2) If $H(A)=H(B) \Rightarrow A=B$.

Definition 4 [49]: An IVq-ROFS is given by:

$$
A=\left\{\left(k,\left[T_{A}^{\prime L}(k), T_{A}^{\prime U}(k)\right],\left[F_{A}^{\prime L}(k), F_{A}^{\prime U}(k)\right]\right): k \in K\right\},
$$

where $T_{A}^{\prime L}, T_{A}^{\prime U}, F_{A}^{\prime L}, F_{A}^{\prime U}: K \rightarrow[0,1]$ represent the lower and upper membership degrees and the lower and upper non-membership degrees, with a condition that $0 \leq T_{A}^{\prime U q}+F_{A}^{\prime U q} \leq 1, q \geq 1$. The IntervalValued q-ROFN (IVq-ROFN) is denoted by $A=$ $\left(\left[T_{A}^{\prime L}, T_{A}^{\prime U}\right],\left[F_{A}^{\prime L}, F_{A}^{\prime \prime}\right]\right)$.

Definition 5 [49]: Let $A=\left(\left[T_{A}^{L}, T_{A}^{\prime U}\right],\left[F_{A}^{\prime L}, F_{A}^{\prime U}\right]\right)$ 
and $B=\left(\left[T_{B}^{\prime L}, T_{B}^{\prime U}\right],\left[F_{B}^{\prime L}, F_{B}^{\prime U}\right]\right)$ be two IVq-ROFNs. Then, we have:

$$
\begin{aligned}
& A^{c}=\left(\left[F_{A}^{\prime L}, F_{A}^{\prime U}\right],\left[T_{A}^{\prime L}, T_{A}^{\prime U}\right]\right) ; \\
& A \vee B=\left(\left[\max \left(T_{A}^{L}, T_{B}^{L}\right), \max \left(T_{A}^{\prime U}, T_{B}^{\prime U}\right)\right]\right. \\
& {\left.\left[\min \left(F_{A}^{\prime L}, F_{B}^{\prime L}\right), \min \left(F_{A}^{\prime U}, F_{B}^{\prime U}\right)\right]\right) ; } \\
& A \wedge B=\left(\left[\min \left(T_{A}^{\prime L}, T_{B}^{\prime L}\right), \min \left(T_{A}^{\prime U}, T_{B}^{\prime U}\right)\right]\right. \\
& {\left.\left[\max \left(F_{A}^{\prime L}, F_{B}^{\prime L}\right), \max \left(F_{A}^{\prime U}, F_{B}^{\prime U}\right)\right]\right) . }
\end{aligned}
$$

Definition 6 [49]: Let $A=\left(\left[T_{A}^{\prime L}, T_{A}^{\prime U}\right],\left[F_{A}^{\prime L}, F_{A}^{\prime U}\right]\right)$ be an IVq-ROFN. Then, the score and accuracy functions of $A$ are given by:

$$
\begin{aligned}
& S(A)=\frac{T_{A}^{\prime L q}+T_{A}^{\prime U q}}{2}-\frac{F_{A}^{\prime L q}+F_{A}^{\prime U q}}{2}, \\
& S(A) \in[-1,1], \\
& H(A)=\frac{T_{A}^{\prime L q}+T_{A}^{\prime U q}}{2}+\frac{F_{A}^{\prime L q}+F_{A}^{\prime U q}}{2},
\end{aligned}
$$$$
H(A) \in[0,1]
$$

Definition 7 [49]: Let $A=\left(\left[T_{A}^{\prime L}, T_{A}^{\prime U}\right],\left[F_{A}^{\prime L}, F_{A}^{\prime U}\right]\right)$ and $B=\left(\left[T_{B}^{\prime L}, T_{B}^{\prime U}\right],\left[F_{B}^{\prime L}, F_{B}^{\prime U}\right]\right)$ be two IVq-ROFNs. Then, the comparison method for IVq-ROFNs $A$ and $B$ is given as follows:

1. If $S(A) \succ S(B) \Rightarrow A \succ B$;

2. If $S(A)=S(B) \Rightarrow$

1) If $H(A) \succ H(B) \Rightarrow A \succ B$;

2) If $H(A)=H(B) \Rightarrow A=B$.

Next, we give the definitions of the existing JSMs [50], DSMs [51], CSMs [52], and VCSMs [53].

Definition 8 [50]: A JSM is given by:

$$
\begin{aligned}
J(K, Y) & =\frac{K Y}{\|K\|^{2}+\|Y\|^{2}-K Y} \\
& =\frac{\sum_{i=1}^{n} k_{i} y_{i}}{\sum_{i=1}^{n} k_{i}^{2}+\sum_{i=1}^{n} y_{i}^{2}-\sum_{i=1}^{n} k_{i} y_{i}},
\end{aligned}
$$

where $K=\left(k_{1}, k_{2}, \cdots, k_{n}\right)$ and $Y=\left(y_{1}, y_{2}, \cdots, y_{n}\right)$ represent two vectors whose Euclidean norm and inner product are followed as $\|K\|=\sqrt{\sum_{i=1}^{n} k_{i}^{2}},\|Y\|=$ $\sqrt{\sum_{i=1}^{n} y_{i}^{2}}$, and $K Y=\sum_{i=1}^{n} k_{i} y_{i}$. Then, Eq. (7) holds the following conditions:

1. $0 \leq J(K, Y) \leq 1$

2. $J(K, Y)=J(Y, K)$;

3. $J(K, Y)=1$ if $K=Y$, i.e., $k_{i}=y_{i}(i=1,2, \cdots, n)$.
Definition 9 [51]: A DSM is given by:

$$
D(K, Y)=\frac{2 K Y}{\|K\|^{2}+\|Y\|^{2}}=\frac{2 \sum_{i=1}^{n} k_{i} y_{i}}{\sum_{i=1}^{n} k_{i}^{2}+\sum_{i=1}^{n} y_{i}^{2}}
$$

where $K=\left(k_{1}, k_{2}, \cdots, k_{n}\right)$ and $Y=\left(y_{1}, y_{2}, \cdots, y_{n}\right)$ represent two vectors whose Euclidean norm and inner product are followed as $\|K\|=\sqrt{\sum_{i=1}^{n} k_{i}^{2}},\|Y\|=$ $\sqrt{\sum_{i=1}^{n} y_{i}^{2}}$. Then, Eq. (8) holds the following conditions:

1. $0 \leq D(K, Y) \leq 1$

2. $D(K, Y)=D(Y, K)$;

3. $D(K, Y)=1$ if $K=Y$ i.e., $k_{i}=y_{i}(i=1,2, \cdots, n)$.

Definition 10 [52]: A CSM is given by:

$$
C(K, Y)=\frac{K Y}{\|K\|+\|Y\|}=\frac{\sum_{i=1}^{n} k_{i} y_{i}}{\sqrt{\sum_{i=1}^{n} k_{i}^{2}}+\sqrt{\sum_{i=1}^{n} y_{i}^{2}}},
$$

where $K=\left(k_{1}, k_{2}, \cdots, k_{n}\right)$ and $Y=\left(y_{1}, y_{2}, \cdots, y_{n}\right)$ represent two vectors whose Euclidean norm and inner product are followed as $\|K\|=\sqrt{\sum_{i=1}^{n} k_{i}^{2}},\|Y\|=$ $\sqrt{\sum_{i=1}^{n} y_{i}^{2}}$. Then, Eq. (9) holds the following conditions:

1. $0 \leq C(K, Y) \leq 1$;

2. $C(K, Y)=C(Y, K)$;

3. $C(K, Y)=1$ if $K=Y$ i.e., $k_{i}=y_{i}(i=1,2, \cdots, n)$.

Definition 11 [53]: A VCSM is given by:

$$
\begin{gathered}
V(K, Y)=\rho \frac{2 K Y}{\|K\|^{2}+\|Y\|^{2}}+(1-\rho) \frac{K Y}{\|K\| \cdot\|Y\|} \\
=\rho \frac{2 \sum_{i=1}^{n} k_{i} y_{i}}{\sum_{i=1}^{n} k_{i}^{2}+\sum_{i=1}^{n} y_{i}^{2}}+(1-\rho) \frac{\sum_{i=1}^{n} k_{i} y_{i}}{\sqrt{\sum_{i=1}^{n} k_{i}^{2}} \cdot \sqrt{\sum_{i=1}^{n} y_{i}^{2}}},
\end{gathered}
$$

where $K=\left(k_{1}, k_{2}, \cdots, k_{n}\right)$ and $Y=\left(y_{1}, y_{2}, \cdots, y_{n}\right)$ represent two vectors whose Euclidean norm and inner product are followed as $\|K\|=\sqrt{\sum_{i=1}^{n} k_{i}^{2}},\|Y\|=$ $\sqrt{\sum_{i=1}^{n} y_{i}^{2}}$, and $K Y=\sum_{i=1}^{n} k_{i} y_{i}$ with $\rho \geq 1$. Then, Eq. (10) holds the following conditions:

1. $0 \leq V(K, Y) \leq 1$

2. $V(K, Y)=V(Y, K)$;

3. $V(K, Y)=1$ if $K=Y$ i.e., $k_{i}=y_{i}(i=1,2, \cdots, n)$.

These four similarity measures come with the same meaning in the domain of interval values. If $k_{i}=0$ and $y_{i}=0$, then the JSMs and DSMs are undefined. If $k_{i}=0$ or $y_{i}=0$, then the CSMs are undefined. 


\subsection{Cq-ROFSs and IVCq-ROFSs}

This subsection proposes the Cq-ROFSs and IVCqROFSs and discusses their basic properties.

Definition 12: A Cq-ROFS is given by:

$$
A=\left\{\left(k, T_{A}^{\prime}(k), F_{A}^{\prime}(k)\right): k \in K\right\},
$$

where $T_{A}^{\prime}=T_{A} e^{i 2 \pi\left(\varphi_{T_{A}}\right)}$ and $F_{A}^{\prime}=F_{A} e^{i 2 \pi\left(\varphi_{F_{A}}\right)}$ represent complex-valued membership and complexvalued non-membership, with a condition that: $0 \leq$ $T_{A}^{q}+F_{A}^{q} \leq 1$, and $0 \leq \varphi_{T_{A}}^{q}+\varphi_{F_{A}}^{q} \leq 1$ for $q \geq 1$. The complex q-ROFN (Cq-ROFN) is denoted by:

$$
A=\left(T_{A} e^{i 2 \pi\left(\varphi_{T_{A}}\right)}, F_{A} e^{i 2 \pi\left(\varphi_{F_{A}}\right)}\right) .
$$

Definition 13: Let $A=\left(T_{A} e^{i 2 \pi\left(\varphi_{T_{A}}\right)}, F_{A} e^{i 2 \pi\left(\varphi_{F_{A}}\right)}\right)$ and $B=\left(T_{B} e^{i 2 \pi\left(\varphi_{T_{B}}\right)}, F_{B} e^{i 2 \pi\left(\varphi_{F_{B}}\right)}\right)$ be two CqROFNs. Then, we have:

$$
\begin{aligned}
A^{c}=\left(F_{A} e^{i 2 \pi\left(\varphi_{F_{A}}\right)}, T_{A} e^{i 2 \pi\left(\varphi_{T_{A}}\right)}\right) ; & \\
A \vee B= & \left(\max \left(T_{A}, T_{B}\right) e^{i 2 \pi\left(\max \left(\varphi_{T_{A}}, \varphi_{T_{B}}\right)\right)},\right. \\
& \left.\min \left(F_{A}, F_{B}\right) e^{i 2 \pi\left(\min \left(\varphi_{F_{A}}, \varphi_{F_{B}}\right)\right)}\right) ; \\
A \wedge B= & \left(\min \left(T_{A}, T_{B}\right) e^{i 2 \pi\left(\min \left(\varphi_{T_{A}}, \varphi_{T_{B}}\right)\right)},\right. \\
& \left.\max \left(F_{A}, F_{B}\right) e^{i 2 \pi\left(\max \left(\varphi_{F_{A}}, \varphi_{F_{B}}\right)\right)}\right) .
\end{aligned}
$$

Definition 14: Let $A=\left(T_{A} e^{i 2 \pi\left(\varphi_{T_{A}}\right)}, F_{A} e^{i 2 \pi\left(\varphi_{F_{A}}\right)}\right)$ be a Cq-ROFN. Then, the score and accuracy functions of $A$ are given by:

$$
\begin{aligned}
& S(A)=\frac{1}{2}\left(T_{A}^{q}+\varphi_{T_{A}}^{q}-\varphi_{F_{A}}^{q}-F_{A}^{q}\right), \\
& S(A) \in[-1,1], \\
& H(A)=\frac{1}{2}\left(T_{A}^{q}+\varphi_{T_{A}}^{q}+\varphi_{F_{A}}^{q}+F_{A}^{q}\right), \\
& H(A) \in[0,1] .
\end{aligned}
$$

Definition 15: Let $A=\left(T_{A} e^{i 2 \pi\left(\varphi_{T_{A}}\right)}, F_{A} e^{i 2 \pi\left(\varphi_{F_{A}}\right)}\right)$ and $B=\left(T_{B} e^{i 2 \pi\left(\varphi_{T_{B}}\right)}, F_{B} e^{i 2 \pi\left(\varphi_{F_{B}}\right)}\right)$ be two CqROFNs. Then, the comparison method for Cq-ROFNs $A$ and $B$ is given as follows:

1. If $S(A) \succ S(B) \Rightarrow A \succ B$;

2. If $S(A)=S(B) \Rightarrow$
1) If $H(A) \succ H(B) \Rightarrow A \succ B$;

2) If $H(A)=H(B) \Rightarrow A=B$.

Example 1: Let $A=\left(0.8 e^{i 2 \pi(0.7)}, 0.9 e^{i 2 \pi(0.67)}\right)$ and $B=\left(0.88 e^{i 2 \pi(0.75)}, 0.67 e^{i 2 \pi(0.66)}\right)$ be two Cq-ROFNs for $q=5$. Then, we have:

$$
\begin{aligned}
A^{c}=\left(0.9 e^{i 2 \pi(0.67)}, 0.8 e^{i 2 \pi(0.7)}\right) & \\
A \vee B= & \left(\max (0.8,0.88) e^{i 2 \pi(\max (0.7,0.75))},\right. \\
& \left.\min (0.9,0.67) e^{i 2 \pi(\min (0.67,0.66))}\right) \\
= & \left(0.88 e^{i 2 \pi(0.75)}, 0.67 e^{i 2 \pi(0.66)}\right) ; \\
A \wedge B= & \left(\min (0.8,0.88) e^{i 2 \pi(\min (0.7,0.75))},\right. \\
& \left.\max (0.9,0.67) e^{i 2 \pi(\max (0.67,0.66))}\right) \\
= & \left(0.88 e^{i 2 \pi(0.7)}, 0.9 e^{i 2 \pi(0.67)}\right) .
\end{aligned}
$$

Next, we calculate the score values of $A$ and $B$ as follows:

$$
\begin{aligned}
S(A) & =\frac{1}{2}\left((0.8)^{5}+(0.7)^{5}-(0.9)^{5}-(0.67)^{5}\right) \\
& =\frac{1}{2}(0.50-0.73)=-0.115, \\
S(B) & =\frac{1}{2}\left((0.88)^{5}+(0.75)^{5}-(0.67)^{5}-(0.66)^{5}\right) \\
& =\frac{1}{2}(0.77-0.26)=0.255 .
\end{aligned}
$$

So, $B \succ A$.

Definition 16: An IVCq-ROFS is given by:

$$
A=\left\{\left(k, T_{A}^{\prime}(k), F_{A}^{\prime}(k)\right): k \in K\right\},
$$

where $T_{A}^{\prime}=\left[T_{A}^{L}, T_{A}^{U}\right] \cdot e^{i 2 \pi\left[\varphi_{T_{A}}^{L}, \varphi_{T_{A}}^{U}\right]}$ and $F_{A}^{\prime}=\left[F_{A}^{L}, F_{A}^{U}\right]$. $e^{i 2 \pi\left[\varphi_{F_{A}}^{L}, \varphi_{F_{A}}^{U}\right]}$ represent interval-valued complex membership and interval-valued complex non-membership, with a condition that $0 \leq T_{A}^{U q}+F_{A}^{U q} \leq 1$ and $0 \leq \varphi_{T_{A}}^{U q}+\varphi_{F_{A}}^{U q} \leq 1, q \geq 1$. The interval-valued CqROFN (IVCq-ROFN) is denoted by $A=\left(\left[T_{A}^{L}, T_{A}^{U}\right]\right.$. $\left.e^{i 2 \pi\left[\varphi_{T_{A}}^{L}, \varphi_{T_{A}}^{U}\right]},\left[F_{A}^{L}, F_{A}^{U}\right] \cdot e^{i 2 \pi\left[\varphi_{F_{A}}^{L}, \varphi_{F_{A}}^{U}\right]}\right)$.

Definition 17: Let $A=\left(\left[T_{A}^{L}, T_{A}^{U}\right] \cdot e^{i 2 \pi\left[\varphi_{T_{A}}^{L}, \varphi_{T_{A}}^{U}\right]},\left[F_{A}^{L}\right.\right.$, $\left.\left.F_{A}^{U}\right] \cdot e^{i 2 \pi\left[\varphi_{F_{A}}^{L}, \varphi_{F_{A}}^{U}\right]}\right)$ and $B=\left(\left[T_{B}^{L}, T_{B}^{U}\right] \cdot e^{i 2 \pi\left[\varphi_{T_{B}}^{L}, \varphi_{T_{B}}^{U}\right]}\right.$, $\left.\left[F_{B}^{L}, F_{B}^{U}\right] \cdot e^{i 2 \pi\left[\varphi_{F_{B}}^{L}, \varphi_{F_{B}}^{U}\right]}\right)$ be two IVCq-ROFNs; then, we have:

$$
\begin{aligned}
A^{c}=\left(\left[F_{A}^{L}, F_{A}^{U}\right] \cdot e^{i 2 \pi\left[\varphi_{F_{A}}^{L}, \varphi_{F_{A}}^{U}\right]},\left[T_{A}^{L}, T_{A}^{U}\right]\right. \\
\left.\cdot e^{i 2 \pi\left[\varphi_{T_{A}}^{L}, \varphi_{T_{A}}^{U}\right]}\right) ; \\
A \vee B=\left(\left[\max \left(T_{A}^{L}, T_{B}^{L}\right), \max \left(T_{A}^{U}, T_{B}^{U}\right)\right]\right. \\
\cdot e^{i 2 \pi\left[\max \left(\varphi_{T_{A}}^{L}, \varphi_{T_{B}}^{L}\right), \max \left(\varphi_{T_{A}}^{U}, \varphi_{T_{B}}^{U}\right)\right]}, \\
{\left[\min \left(F_{A}^{L}, F_{B}^{L}\right), \min \left(F_{A}^{U}, F_{B}^{U}\right)\right] } \\
\left.\cdot e^{i 2 \pi\left[\min \left(\varphi_{F_{A}}^{L}, \varphi_{F_{B}}^{L}\right), \min \left(\varphi_{F_{A}}^{U}, \varphi_{F_{B}}^{U}\right)\right]}\right) ;
\end{aligned}
$$




$$
\begin{aligned}
A \wedge B= & \left(\left[\min \left(T_{A}^{L}, T_{B}^{L}\right), \min \left(T_{A}^{U}, T_{B}^{U}\right)\right]\right. \\
& \cdot e^{i 2 \pi\left[\min \left(\varphi_{T_{A}}^{L}, \varphi_{T_{B}}^{L}\right), \min \left(\varphi_{T_{A}}^{U}, \varphi_{T_{B}}^{U}\right)\right]}, \\
& {\left[\max \left(F_{A}^{L}, F_{B}^{L}\right), \max \left(F_{A}^{U}, F_{B}^{U}\right)\right] } \\
& \left.\cdot e^{i 2 \pi\left[\max \left(\varphi_{F_{A}}^{L}, \varphi_{F_{B}}^{L}\right), \max \left(\varphi_{F_{A}}^{U}, \varphi_{F_{B}}^{U}\right)\right]}\right) .
\end{aligned}
$$

Definition 18: Let $A=\left(\left[T_{A}^{L}, T_{A}^{U}\right] \cdot e^{i 2 \pi\left[\varphi_{T_{A}}^{L}, \varphi_{T_{A}}^{U}\right]}\right.$, $\left.\left[F_{A}^{L}, F_{A}^{U}\right] \cdot e^{i 2 \pi\left[\varphi_{F_{A}}^{L}, \varphi_{F_{A}}^{U}\right]}\right)$ be an IVCq-ROFN. Then, the score and accuracy functions of $A$ are given by:

$$
\begin{aligned}
S(A)= & \frac{1}{2}\left(\frac{T_{A}^{L}+T_{A}^{U}}{2}+\frac{\varphi_{T_{A}}^{L}+\varphi_{T_{A}}^{U}}{2}\right. \\
& \left.-\frac{F_{A}^{L}+F_{A}^{U}}{2}-\frac{\varphi_{F_{A}}^{L}+\varphi_{F_{A}}^{U}}{2}\right), \quad S(A) \in[-1,1], \\
H(A) & =\frac{1}{2}\left(\frac{T_{A}^{L}+T_{A}^{U}}{2}+\frac{\varphi_{T_{A}}^{L}+\varphi_{T_{A}}^{U}}{2}\right. \\
& \left.+\frac{F_{A}^{L}+F_{A}^{U}}{2}+\frac{\varphi_{F_{A}}^{L}+\varphi_{F_{A}}^{U}}{2}\right), \quad H(A) \in[0,1] .
\end{aligned}
$$

Definition 19: Let $A=\left(\left[T_{A}^{L}, T_{A}^{U}\right] \cdot e^{i 2 \pi\left[\varphi_{T_{A}}^{L}, \varphi_{T_{A}}^{U}\right]},\left[F_{A}^{L}\right.\right.$, $\left.\left.F_{A}^{U}\right] \cdot e^{i 2 \pi\left[\varphi_{F_{A}}^{L}, \varphi_{F_{A}}^{U}\right]}\right)$ and $B=\left(\left[T_{B}^{L}, T_{B}^{U}\right] \cdot e^{i 2 \pi\left[\varphi_{T_{B}}^{L}, \varphi_{T_{B}}^{U}\right]}\right.$, $\left.\left[F_{B}^{L}, F_{B}^{U}\right] \cdot e^{i 2 \pi\left[\varphi_{F_{B}}^{L}, \varphi_{F_{B}}^{U}\right]}\right)$ be two IVCq-ROFNs. Then, the comparison method for IVCq-ROFNs $A$ and $B$ is given as follows:

1. If $S(A) \succ S(B) \Rightarrow A \succ B$;

2. If $S(A)=S(B) \Rightarrow$

1) If $H(A) \succ H(B) \Rightarrow A \succ B$;

2) If $H(A)=H(B) \Rightarrow A=B$.

Example 2: $\quad$ Let $A=\left([0.8,0.82] e^{i 2 \pi[0.7,0.72]},[0.9,0.92]\right.$ $\left.e^{i 2 \pi[0.67,0.69]}\right)$ and $B=\left([0.88,0.9] e^{i 2 \pi[0.75,0.77]},[0.67\right.$, $\left.0.69] e^{i 2 \pi[0.66, .0 .68]}\right)$ be two Cq-ROFNs for $q=5$. Then, we have:

$$
\begin{aligned}
A^{c}=\left([0.9,0.92] e^{i 2 \pi[0.67,0.69]},[0.8,0.82] e^{i 2 \pi[0.7,0.72]}\right) & \\
A \vee B= & ([\max (0.8,0.88), \max (0.82,0.9)] \\
& e^{i 2 \pi[\max (0.7,0.75), \max (0.72,0.77)]},[\min (0.9,0.67) \\
& \left.\min (0.92,0.69)] e^{i 2 \pi[\min (0.67,0.66), \min (0.69,0,68)}\right) \\
& =\left([0.88,0.9] e^{i 2 \pi[0.75,0.77]}\right. \\
& {\left.[0.67,0.69] e^{i 2 \pi[0.66, .0 .68]}\right) }
\end{aligned}
$$

$A \wedge B=([\min (0.8,0.88), \min (0.82,0.9)]$

$$
\begin{aligned}
& e^{i 2 \pi[\min (0.7,0.75), \min (0.72,0.77)]},[\max (0.9,0.67) \\
& \left.\max (0.92,0.69)] e^{i 2 \pi[\max (0.67,0.66), \max (0.69,0,68)}\right) \\
& =\left([0.8,0.82] e^{i 2 \pi[0.7,0.72]}\right. \\
& \left.[0.9,0.92] e^{i 2 \pi[0.67, .0 .69]}\right)
\end{aligned}
$$

Next, we calculate the score values of $A$ and $B$ and get

$$
\begin{aligned}
S(A)= & \frac{1}{2}\left(\left((0.8)^{5}+(0.82)^{5}\right) / 2+\left((0.7)^{5}+(0.72)^{5}\right) / 2\right. \\
& \left.-\left((0.9)^{5}+(0.92)^{5}\right) / 2-\left((0.67)^{5}+(0.69)^{5}\right) / 2\right) \\
= & \frac{1}{2}(0.35+0.18-0.62+0.15)=-0.12, \\
S(B)= & \frac{1}{2}\left(\left((0.88)^{5}+(0.9)^{5}\right) / 2+\left((0.75)^{5}+(0.77)^{5}\right) / 2\right. \\
& \left.-\left((0.67)^{5}+(0.69)^{5}\right) / 2-\left((0.66)^{5}+(0.68)^{5}\right) / 2\right) \\
= & \frac{1}{2}(0.56+0.25-0.15+0.14)=0.2 .
\end{aligned}
$$

So, $B \succ A$.

\section{Hybrid vector similarity measures for Cq-ROFSs}

This section investigates some VSMs called Jaccard, Dice, and cosine similarity measures and HVSM called variation coefficient similarity measures for Cq-ROFSs. For convenience, in this paper, the weighted vector is defined by: $\omega=\left\{\omega_{1}, \omega_{2}, \cdots, \omega_{n}\right\}^{T}, \omega_{i} \in[0,1]$, $i=1,2, \cdots, n$ with a condition that $\sum_{i=1}^{n} \omega_{i}=1$. In addition, $A=\left(T_{A} e^{i 2 \pi\left(\varphi_{T_{A}}\right)}, F_{A} e^{i 2 \pi\left(\varphi_{F_{A}}\right)}\right)$ and $B=$ $\left(T_{B} e^{i 2 \pi\left(\varphi_{T_{B}}\right)}, F_{B} e^{i 2 \pi\left(\varphi_{F_{B}}\right)}\right)$ are used to denote the CqROFNs.

\subsection{The VSMs for Cq-ROFSs}

The Jaccard, Dice, and cosine similarity measures are important tools to find the degree of similarity between objects. In this subsection, we extend them to CqROFSs and propose JSM, Weighted JSM (WJSM), DSM, Weighted DSM (WDSM), CSM, and Weighted CSM (WCSM) for Cq-ROFSs.

Definition 20: The JSM for Cq-ROFS is given by Eq. (17) as shown in Box I. Eq. (17) holds the following conditions:

1. $0 \leq J_{j c}(A, B) \leq 1$,

2. $J_{j c}(A, B)=J_{j c}(B, A)$,

3. $J_{j c}(A, B)=1$, for $A=B$ i.e., $T_{A}=T_{B}, \varphi_{T_{A}}=$ $\varphi_{T_{B}}, F_{A}=F_{B}$, and $\varphi_{F_{A}}=\varphi_{F_{B}}$. 


$$
J_{j c}(A, B)=\frac{1}{2 n} \sum_{i=1}^{n}\left(\begin{array}{l}
\frac{T_{A}^{q}\left(k_{i}\right) T_{B}^{q}\left(k_{i}\right)+F_{A}^{q}\left(k_{i}\right) F_{B}^{q}\left(k_{i}\right)}{\left(T_{A}^{2 q}\left(k_{i}\right)+F_{A}^{2 q}\left(k_{i}\right)\right)+\left(T_{B}^{2 q}\left(k_{i}\right)+F_{B}^{2 q}\left(k_{i}\right)\right)-\left(T_{A}^{q}\left(k_{i}\right) T_{B}^{q}\left(k_{i}\right)+F_{A}^{q}\left(k_{i}\right) F_{B}^{q}\left(k_{i}\right)\right)} \\
+\frac{\varphi_{T_{A}}^{q}\left(k_{i}\right) \varphi_{T_{B}}^{q}\left(k_{i}\right)+\varphi_{F_{A}}^{q}\left(k_{i}\right) \varphi_{F_{B}}^{q}\left(k_{i}\right)}{\left(\varphi_{T_{A}}^{2 q}\left(k_{i}\right)+\varphi_{F_{A}}^{2 q}\left(k_{i}\right)\right)+\left(\varphi_{T_{B}}^{2 q}\left(k_{i}\right)+\varphi_{F_{B}}^{2 q}\left(k_{i}\right)\right)-\left(\varphi_{T_{A}}^{q}\left(k_{i}\right) \varphi_{T_{B}}^{q}\left(k_{i}\right)+\varphi_{F_{A}}^{q}\left(k_{i}\right) \varphi_{F_{B}}^{q}\left(k_{i}\right)\right)}
\end{array}\right) .
$$

Box I

$$
J_{w j c}(A, B)=\frac{1}{2} \sum_{i=1}^{n} \omega_{i}\left(\begin{array}{l}
\frac{T_{A}^{q}\left(k_{i}\right) T_{B}^{q}\left(k_{i}\right)+F_{A}^{q}\left(k_{i}\right) F_{B}^{q}\left(k_{i}\right)}{\left(T_{A}^{2 q}\left(k_{i}\right)+F_{A}^{2 q}\left(k_{i}\right)\right)+\left(T_{B}^{2 q}\left(k_{i}\right)+F_{B}^{2 q}\left(k_{i}\right)\right)-\left(T_{A}^{q}\left(k_{i}\right) T_{B}^{q}\left(k_{i}\right)+F_{A}^{q}\left(k_{i}\right) F_{B}^{q}\left(k_{i}\right)\right)} \\
+\frac{\varphi_{T_{A}}^{q}\left(k_{i}\right) \varphi_{T_{B}}^{q}\left(k_{i}\right)+\varphi_{F_{A}}^{q}\left(k_{i}\right) \varphi_{F_{B}}^{q}\left(k_{i}\right)}{\left(\varphi_{T_{A}}^{2 q}\left(k_{i}\right)+\varphi_{F_{A}}^{2 q}\left(k_{i}\right)\right)+\left(\varphi_{T_{B}}^{2 q}\left(k_{i}\right)+\varphi_{F_{B}}^{2 q}\left(k_{i}\right)\right)-\left(\varphi_{T_{A}}^{q}\left(k_{i}\right) \varphi_{T_{B}}^{q}\left(k_{i}\right)+\varphi_{F_{A}}^{q}\left(k_{i}\right) \varphi_{F_{B}}^{q}\left(k_{i}\right)\right)}
\end{array}\right) ;
$$

\section{Box II}

Definition 21: The WJSM for Cq-ROFS is given by Eq. (18) as shown in Box II. Eq. (18) holds the following conditions:

1. $0 \leq J_{w j c}(A, B) \leq 1$,

2. $J_{w j c}(A, B)=J_{w j c}(B, A)$,

3. $J_{w j c}(A, B)=1$, for $A=B$ i.e., $T_{A}=T_{B}, \varphi_{T_{A}}=$ $\varphi_{T_{B}}, F_{A}=F_{B}$, and $\varphi_{F_{A}}=\varphi_{F_{B}}$.

Definition 22: The DSM for Cq-ROFS is given by Eq. (19) as shown in Box III. Eq. (19) holds the following conditions:

1. $0 \leq J_{d}(A, B) \leq 1$,
2. $J_{d}(A, B)=J_{d}(B, A)$,

3. $J_{d}(A, B)=1$, for $A=B$, i.e., $T_{A}=T_{B}, \varphi_{T_{A}}=$ $\varphi_{T_{B}}, F_{A}=F_{B}$, and $\varphi_{F_{A}}=\varphi_{F_{B}}$.

Definition 23: The WDSM for Cq-ROFS is given by Eq. (20) as shown in Box IV. Eq. (20) holds the following conditions

1. $0 \leq J_{W d}(A, B) \leq 1$,

2. $J_{W d}(A, B)=J_{W d}(B, A)$,

3. $J_{W d}(A, B)=1$, for $A=B$ i.e., $T_{A}=T_{B}, \varphi_{T_{A}}=$ $\varphi_{T_{B}}, F_{A}=F_{B}$, and $\varphi_{F_{A}}=\varphi_{F_{B}}$.

$$
J_{d}(A, B)=\frac{1}{2 n} \sum_{i=1}^{n}\left(\begin{array}{l}
\frac{2\left(T_{A}^{q}\left(k_{i}\right) T_{B}^{q}\left(k_{i}\right)+F_{A}^{q}\left(k_{i}\right) F_{B}^{q}\left(k_{i}\right)\right)}{\left(T_{A}^{2 q}\left(k_{i}\right)+F_{A}^{2 q}\left(k_{i}\right)\right)+\left(T_{B}^{2 q}\left(k_{i}\right)+F_{B}^{2 q}\left(k_{i}\right)\right)} \\
+\frac{2\left(\varphi_{T_{A}}^{q}\left(k_{i}\right) \varphi_{T_{B}}^{q}\left(k_{i}\right)+\varphi_{F_{A}}^{q}\left(k_{i}\right) \varphi_{F_{B}}^{q}\left(k_{i}\right)\right)}{\left(\varphi_{T_{A}}^{2 q}\left(k_{i}\right)+\varphi_{F_{A}}^{2 q}\left(k_{i}\right)\right)+\left(\varphi_{T_{B}}^{2 q}\left(k_{i}\right)+\varphi_{F_{B}}^{2 q}\left(k_{i}\right)\right)}
\end{array}\right) .
$$

\section{Box III}

$$
J_{W d}(A, B)=\frac{1}{2} \sum_{i=1}^{n} \omega_{i}\left(\begin{array}{l}
\frac{2\left(T_{A}^{q}\left(k_{i}\right) T_{B}^{q}\left(k_{i}\right)+F_{A}^{q}\left(k_{i}\right) F_{B}^{q}\left(k_{i}\right)\right)}{\left(T_{A}^{2 q}\left(k_{i}\right)+F_{A}^{2 q}\left(k_{i}\right)\right)+\left(T_{B}^{2 q}\left(k_{i}\right)+F_{B}^{2 q}\left(k_{i}\right)\right)} \\
+\frac{2\left(\varphi_{T_{A}}^{q}\left(k_{i}\right) \varphi_{T_{B}}^{q}\left(k_{i}\right)+\varphi_{F_{A}}^{q}\left(k_{i}\right) \varphi_{F_{B}}^{q}\left(k_{i}\right)\right)}{\left(\varphi_{T_{A}}^{2 q}\left(k_{i}\right)+\varphi_{F_{A}}^{2 q}\left(k_{i}\right)\right)+\left(\varphi_{T_{B}}^{2 q}\left(k_{i}\right)+\varphi_{F_{B}}^{2 q}\left(k_{i}\right)\right)}
\end{array}\right) .
$$




$$
J_{c}(A, B)=\frac{1}{2 n} \sum_{i=1}^{n}\left(\begin{array}{l}
\frac{2\left(T_{A}^{q}\left(k_{i}\right) T_{B}^{q}\left(k_{i}\right)+F_{A}^{q}\left(k_{i}\right) F_{B}^{q}\left(k_{i}\right)\right)}{\sqrt{\left(T_{A}^{2 q}\left(k_{i}\right)+F_{A}^{2 q}\left(k_{i}\right)\right)} \cdot \sqrt{\left(T_{B}^{2 q}\left(k_{i}\right)+F_{B}^{2 q}\left(k_{i}\right)\right)}} \\
+\frac{2\left(\varphi_{T_{A}}^{q}\left(k_{i}\right) \varphi_{T_{B}}^{q}\left(k_{i}\right)+\varphi_{F_{A}}^{q}\left(k_{i}\right) \varphi_{F_{B}}^{q}\left(k_{i}\right)\right)}{\sqrt{\left(\varphi_{T_{A}}^{2 q}\left(k_{i}\right)+\varphi_{F_{A}}^{2 q}\left(k_{i}\right)\right)} \cdot \sqrt{\left(\varphi_{T_{B}}^{2 q}\left(k_{i}\right)+\varphi_{F_{B}}^{2 q}\left(k_{i}\right)\right)}}
\end{array}\right) .
$$

Box V

$$
J_{W c}(A, B)=\frac{1}{2} \sum_{i=1}^{n} \omega_{i}\left(\begin{array}{l}
\frac{2\left(T_{A}^{q}\left(k_{i}\right) T_{B}^{q}\left(k_{i}\right)+F_{A}^{q}\left(k_{i}\right) F_{B}^{q}\left(k_{i}\right)\right)}{\sqrt{\left(T_{A}^{2 q}\left(k_{i}\right)+F_{A}^{2 q}\left(k_{i}\right)\right)} \cdot \sqrt{\left(T_{B}^{2 q}\left(k_{i}\right)+F_{B}^{2 q}\left(k_{i}\right)\right)}} \\
+\frac{2\left(\varphi_{T_{A}}^{q}\left(k_{i}\right) \varphi_{T_{B}}^{q}\left(k_{i}\right)+\varphi_{F_{A}}^{q}\left(k_{i}\right) \varphi_{F_{B}}^{q}\left(k_{i}\right)\right)}{\sqrt{\left(\varphi_{T_{A}}^{2 q}\left(k_{i}\right)+\varphi_{F_{A}}^{2 q}\left(k_{i}\right)\right)} \cdot \sqrt{\left(\varphi_{T_{B}}^{2 q}\left(k_{i}\right)+\varphi_{F_{B}}^{2 q}\left(k_{i}\right)\right)}}
\end{array}\right)
$$

\section{Box VI}

Definition 24: The CSM for Cq-ROFS is given by Eq. (21) as shown in Box V. Eq. (21) holds the following conditions:

1. $0 \leq J_{c}(A, B) \leq 1$,

2. $J_{c}(A, B)=J_{c}(B, A)$,

3. $J_{c}(A, B)=1$, for $A=B$ i.e., $T_{A}=T_{B}, \varphi_{T_{A}}=\varphi_{T_{B}}$, $F_{A}=F_{B}$, and $\varphi_{F_{A}}=\varphi_{F_{B}}$.

Definition 25: The WCSM for Cq-ROFS is given by Eq. (22) as shown in Box VI. Eq. (22) holds the following conditions:

1. $0 \leq J_{W c}(A, B) \leq 1$,

2. $J_{W c}(A, B)=J_{W c}(B, A)$,

3. $J_{W c}(A, B)=1$, for $A=B$ i.e., $T_{A}=T_{B}, \varphi_{T_{A}}=$ $\varphi_{T_{B}}, F_{A}=F_{B}$, and $\varphi_{F_{A}}=\varphi_{F_{B}}$.

\subsection{The VSMs for IVCq-ROFSs}

This subsection combines Jaccard, Dice, and cosine similarity measures with IVCq-ROFS and proposes JSM, WJSM, DSM, WDSM, CSM, and WCSM for IVCq-ROFSs. For convenience, the membership and non-membership values in the domain of IVCq-ROFSs are given as follows:

$$
\begin{aligned}
& 2 \Omega T_{A}^{q}\left(k_{i}\right)=\left(T_{A}^{L}\left(k_{i}\right)+T_{A}^{U}\left(k_{i}\right)\right) / 2, \\
& 2 \Omega F_{A}^{q}\left(k_{i}\right)=\left(F_{A}^{L}\left(k_{i}\right)+F_{A}^{U}\left(k_{i}\right)\right) / 2, \\
& 2 \Omega \varphi_{T_{A}}^{q}\left(k_{i}\right)=\left(\varphi_{T_{A}}^{L}\left(k_{i}\right)+\varphi_{T_{A}}^{U}\left(k_{i}\right)\right) / 2, \\
& 2 \Omega \varphi_{F_{A}}^{q}\left(k_{i}\right)=\left(\varphi_{F_{A}}^{L}\left(k_{i}\right)+\varphi_{F_{A}}^{U}\left(k_{i}\right)\right) / 2,
\end{aligned}
$$

and:

$$
\begin{aligned}
& 2 \Omega T_{B}^{q}\left(k_{i}\right)=\left(T_{B}^{L}\left(k_{i}\right)+T_{B}^{U}\left(k_{i}\right)\right) / 2, \\
& 2 \Omega F_{B}^{q}\left(k_{i}\right)=\left(F_{B}^{L}\left(k_{i}\right)+F_{B}^{U}\left(k_{i}\right)\right) / 2, \\
& 2 \Omega \varphi_{T_{B}}^{q}\left(k_{i}\right)=\left(\varphi_{T_{B}}^{L}\left(k_{i}\right)+\varphi_{T_{B}}^{U}\left(k_{i}\right)\right) / 2, \\
& 2 \Omega \varphi_{F_{B}}^{q}\left(k_{i}\right)=\left(\varphi_{F_{B}}^{L}\left(k_{i}\right)+\varphi_{F_{B}}^{U}\left(k_{i}\right)\right) / 2 .
\end{aligned}
$$

Then, we propose the following similarity measures.

Definition 26: The JSM for IVCq-ROFS is given by Eq. (23) as shown in Box VII. Eq. (23) holds the following conditions:

1. $0 \leq J_{j c}^{I V}(A, B) \leq 1$,

2. $J_{j c}^{I V}(A, B)=J_{j c}^{I V}(B, A)$,

3. $J_{j c}^{I V}(A, B)=1$, for $A=B$ i.e., $T_{A}^{L}=T_{B}^{L}, T_{A}^{U}=T_{B}^{U}$, $F_{A}^{L}=F_{B}^{L}, F_{A}^{U}=F_{B}^{U}, \varphi_{T_{A}}^{L}=\varphi_{T_{B}}^{L}, \varphi_{T_{A}}^{U}=\varphi_{T_{B}}^{U}$, $\varphi_{F_{A}}^{L}=\varphi_{F_{B}}^{L}$, and $\varphi_{F_{A}}^{U}=\varphi_{F_{B}}^{U}$.

Definition 27: The WJSM for IVCq-ROFS is given by Eq. (24) as shown in Box VIII. Eq. (24) holds the following conditions:

1. $0 \leq J_{W j c}^{I V}(A, B) \leq 1$,

2. $J_{W j c}^{I V}(A, B)=J_{W j c}^{I V}(B, A)$,

3. $J_{W j c}^{I V}(A, B)=1$, for $A=B$ i.e., $T_{A}^{L}=T_{B}^{L}, T_{A}^{U}=$ $T_{B}^{U}, F_{A}^{L}=F_{B}^{L}, F_{A}^{U}=F_{B}^{U}, \varphi_{T_{A}}^{L}=\varphi_{T_{B}}^{L}, \varphi_{T_{A}}^{U}=\varphi_{T_{B}}^{U}$, $\varphi_{F_{A}}^{L}=\varphi_{F_{B}}^{L}$, and $\varphi_{F_{A}}^{U}=\varphi_{F_{B}}^{U}$. 


$$
\begin{aligned}
J_{j c}^{I V}(A, B)= & \frac{1}{2 n} \sum_{i=1}^{n} \\
& \left(\begin{array}{l}
\frac{\Omega T_{A}^{q}\left(k_{i}\right) \Omega T_{B}^{q}\left(k_{i}\right)+\Omega F_{A}^{q}\left(k_{i}\right) \Omega F_{B}^{q}\left(k_{i}\right)}{\left(\left(\Omega T_{A}^{q}\left(k_{i}\right)\right)^{2}+\left(\Omega F_{A}^{q}\left(k_{i}\right)\right)^{2}\right)+\left(\left(\Omega T_{B}^{q}\left(k_{i}\right)\right)^{2}+\left(\Omega F_{B}^{q}\left(k_{i}\right)\right)^{2}\right)-\left(\begin{array}{c}
\Omega T_{A}^{q}\left(k_{i}\right) \Omega T_{B}^{q}\left(k_{i}\right) \\
+\Omega F_{A}^{q}\left(k_{i}\right) \Omega F_{B}^{q}\left(k_{i}\right)
\end{array}\right)} \\
+\frac{\Omega \varphi_{T_{A}}^{q}\left(k_{i}\right) \Omega \varphi_{T_{B}}^{q}\left(k_{i}\right)+\Omega \varphi_{F_{A}}^{q}\left(k_{i}\right) \Omega \varphi_{F_{B}}^{q}\left(k_{i}\right)}{\left(\left(\Omega \varphi_{T_{A}}^{q}\left(k_{i}\right)\right)^{2}+\left(\Omega \varphi_{F_{A}}^{q}\left(k_{i}\right)\right)^{2}\right)+\left(\left(\Omega \varphi_{T_{B}}^{q}\left(k_{i}\right)\right)^{2}+\left(\Omega \varphi_{F_{B}}^{q}\left(k_{i}\right)\right)^{2}\right)-\left(\begin{array}{c}
\Omega \varphi_{T_{A}}^{q}\left(k_{i}\right) \Omega \varphi_{T_{B}}^{q}\left(k_{i}\right) \\
+\Omega \varphi_{F_{A}}^{q}\left(k_{i}\right) \Omega \varphi_{F_{B}}^{q}\left(k_{i}\right)
\end{array}\right)}
\end{array}\right) .
\end{aligned}
$$

Box VII

$$
\begin{aligned}
J_{W j c}^{I V}(A, B)= & \frac{1}{2} \sum_{i=1}^{n} \omega_{I} \\
& \left(\begin{array}{l}
\frac{\left(\Omega T_{A}^{q}\left(k_{i}\right) \Omega T_{B}^{q}\left(k_{i}\right)+\Omega F_{A}^{q}\left(k_{i}\right) \Omega F_{B}^{q}\left(k_{i}\right)\right.}{\left.\left(\Omega T_{A}^{q}\left(k_{i}\right)\right)^{2}+\left(\Omega F_{A}^{q}\left(k_{i}\right)\right)^{2}\right)+\left(\left(\Omega T_{B}^{q}\left(k_{i}\right)\right)^{2}+\left(\Omega F_{B}^{q}\left(k_{i}\right)\right)^{2}\right)-\left(\begin{array}{c}
\Omega T_{A}^{q}\left(k_{i}\right) \Omega T_{B}^{q}\left(k_{i}\right) \\
+\Omega F_{A}^{q}\left(k_{i}\right) \Omega F_{B}^{q}\left(k_{i}\right)
\end{array}\right)} \\
\left.+\frac{\Omega \varphi_{T_{A}}^{q}\left(k_{i}\right) \Omega \varphi_{T_{B}}^{q}\left(k_{i}\right)+\Omega \varphi_{F_{A}}^{q}\left(k_{i}\right) \Omega \varphi_{F_{B}}^{q}\left(k_{i}\right)}{\left(\left(\Omega \varphi_{T_{A}}^{q}\left(k_{i}\right)\right)^{2}+\left(\Omega \varphi_{F_{A}}^{q}\left(k_{i}\right)\right)^{2}\right)+\left(\left(\Omega \varphi_{T_{B}}^{q}\left(k_{i}\right)\right)^{2}+\left(\Omega \varphi_{F_{B}}^{q}\left(k_{i}\right)\right)^{2}\right)-\left(\begin{array}{l}
\Omega \varphi_{T_{A}}^{q}\left(k_{i}\right) \Omega \varphi_{T_{B}}^{q}\left(k_{i}\right) \\
+\Omega \varphi_{F_{A}}^{q}\left(k_{i}\right) \Omega \varphi_{F_{B}}^{q}\left(k_{i}\right)
\end{array}\right)}\right)
\end{array}\right)
\end{aligned}
$$

Box VIII

Definition 28: The DSM for IVCq-ROFS is given by Eq. (25) as shown in Box IX. Eq. (25) holds the following conditions:

1. $0 \leq J_{d}^{I V}(A, B) \leq 1$,

2. $J_{d}^{I V}(A, B)=J_{d}^{I V}(B, A)$,

3. $J_{d}^{I V}(A, B)=1$, for $A=B$ i.e., $T_{A}^{L}=T_{B}^{L}, T_{A}^{U}=T_{B}^{U}$, $F_{A}^{L}=F_{B}^{L}, F_{A}^{U}=F_{B}^{U}, \varphi_{T_{A}}^{L}=\varphi_{T_{B}}^{L}, \varphi_{T_{A}}^{U}=\varphi_{T_{B}}^{U}$, $\varphi_{F_{A}}^{L}=\varphi_{F_{B}}^{L}$, and $\varphi_{F_{A}}^{U}=\varphi_{F_{B}}^{U}$.
Definition 29: The WDSM for IVCq-ROFS is given by Eq. (26) as shown in Box X. Eq. (26) holds the following conditions:

1. $0 \leq J_{W d}^{I V}(A, B) \leq 1$,

2. $J_{W d}^{I V}(A, B)=J_{W d}^{I V}(B, A)$,

3. $J_{W d}^{I V}(A, B)=1$, for $A=B$ i.e., $T_{A}^{L}=T_{B}^{L}, T_{A}^{U}=$ $T_{B}^{U}, F_{A}^{L}=F_{B}^{L}, F_{A}^{U}=F_{B}^{U}, \varphi_{T_{A}}^{L}=\varphi_{T_{B}}^{L}, \varphi_{T_{A}}^{U}=\varphi_{T_{B}}^{U}$, $\varphi_{F_{A}}^{L}=\varphi_{F_{B}}^{L}$, and $\varphi_{F_{A}}^{U}=\varphi_{F_{B}}^{U}$.

$$
J_{d}^{I V}(A, B)=\frac{1}{2 n} \sum_{i=1}^{n}\left(\begin{array}{l}
\frac{2\left(\Omega T_{A}^{q}\left(k_{i}\right) \Omega T_{B}^{q}\left(k_{i}\right)+\Omega F_{A}^{q}\left(k_{i}\right) \Omega F_{B}^{q}\left(k_{i}\right)\right)}{\left(\left(\Omega T_{A}^{q}\left(k_{i}\right)\right)^{2}+\left(\Omega F_{A}^{q}\left(k_{i}\right)\right)^{2}\right)+\left(\left(\Omega T_{B}^{q}\left(k_{i}\right)\right)^{2}+\left(\Omega F_{B}^{q}\left(k_{i}\right)\right)^{2}\right)} \\
+\frac{2\left(\Omega \varphi_{T_{A}}^{q}\left(k_{i}\right) \Omega \varphi_{T_{B}}^{q}\left(k_{i}\right)+\Omega \varphi_{F_{A}}^{q}\left(k_{i}\right) \Omega \varphi_{F_{B}}^{q}\left(k_{i}\right)\right)}{\left(\left(\Omega \varphi_{T_{A}}^{q}\left(k_{i}\right)\right)^{2}+\left(\Omega \varphi_{F_{A}}^{q}\left(k_{i}\right)\right)^{2}\right)+\left(\left(\Omega \varphi_{T_{B}}^{q}\left(k_{i}\right)\right)^{2}+\left(\Omega \varphi_{F_{B}}^{q}\left(k_{i}\right)\right)^{2}\right)}
\end{array}\right) .
$$




$$
J_{W d}^{I V}(A, B)=\frac{1}{2} \sum_{i=1}^{n} \omega_{i}\left(\begin{array}{l}
\frac{2\left(\Omega T_{A}^{q}\left(k_{i}\right) \Omega T_{B}^{q}\left(k_{i}\right)+\Omega F_{A}^{q}\left(k_{i}\right) \Omega F_{B}^{q}\left(k_{i}\right)\right)}{\left(\left(\Omega T_{A}^{q}\left(k_{i}\right)\right)^{2}+\left(\Omega F_{A}^{q}\left(k_{i}\right)\right)^{2}\right)+\left(\left(\Omega T_{B}^{q}\left(k_{i}\right)\right)^{2}+\left(\Omega F_{B}^{q}\left(k_{i}\right)\right)^{2}\right)} \\
+\frac{2\left(\Omega \varphi_{T_{A}}^{q}\left(k_{i}\right) \Omega \varphi_{T_{B}}^{q}\left(k_{i}\right)+\Omega \varphi_{F_{A}}^{q}\left(k_{i}\right) \Omega \varphi_{F_{B}}^{q}\left(k_{i}\right)\right)}{\left(\left(\Omega \varphi_{T_{A}}^{q}\left(k_{i}\right)\right)^{2}+\left(\Omega \varphi_{F_{A}}^{q}\left(k_{i}\right)\right)^{2}\right)+\left(\left(\Omega \varphi_{T_{B}}^{q}\left(k_{i}\right)\right)^{2}+\left(\Omega \varphi_{F_{B}}^{q}\left(k_{i}\right)\right)^{2}\right)}
\end{array}\right)
$$

Box X

$$
J_{c}^{I V}(A, B)=\frac{1}{2 n} \sum_{i=1}^{n}\left(\begin{array}{l}
\frac{\left(\Omega T_{A}^{q}\left(k_{i}\right) \Omega T_{B}^{q}\left(k_{i}\right)+\Omega F_{A}^{q}\left(k_{i}\right) \Omega F_{B}^{q}\left(k_{i}\right)\right)}{\sqrt{\left(\left(\Omega T_{A}^{q}\left(k_{i}\right)\right)^{2}+\left(\Omega F_{A}^{q}\left(k_{i}\right)\right)^{2}\right)} \cdot \sqrt{\left(\left(\Omega T_{B}^{q}\left(k_{i}\right)\right)^{2}+\left(\Omega F_{B}^{q}\left(k_{i}\right)\right)^{2}\right)}} \\
+\frac{\left(\Omega \varphi_{T_{A}}^{q}\left(k_{i}\right) \Omega \varphi_{T_{B}}^{q}\left(k_{i}\right)+\Omega \varphi_{F_{A}}^{q}\left(k_{i}\right) \Omega \varphi_{F_{B}}^{q}\left(k_{i}\right)\right)}{\sqrt{\left(\left(\Omega \varphi_{T_{A}}^{q}\left(k_{i}\right)\right)^{2}+\left(\Omega \varphi_{F_{A}}^{q}\left(k_{i}\right)\right)^{2}\right)} \cdot \sqrt{\left(\left(\Omega \varphi_{T_{B}}^{q}\left(k_{i}\right)\right)^{2}+\left(\Omega \varphi_{F_{B}}^{q}\left(k_{i}\right)\right)^{2}\right)}}
\end{array}\right)
$$

Box XI

Definition 30: The CSM for IVCq-ROFS is given by Eq. (27) as shown in Box XI. Eq. (27) holds the following conditions:

1. $0 \leq J_{c}^{I V}(A, B) \leq 1$,

2. $J_{c}^{I V}(A, B)=J_{c}^{I V}(B, A)$,

3. $J_{c}^{I V}(A, B)=1$, for $A=B$ i.e., $T_{A}^{L}=T_{B}^{L}, T_{A}^{U}=T_{B}^{U}$, $F_{A}^{L}=F_{B}^{L}, F_{A}^{U}=F_{B}^{U}, \varphi_{T_{A}}^{L}=\varphi_{T_{B}}^{L}, \varphi_{T_{A}}^{U}=\varphi_{T_{B}}^{U}$, $\varphi_{F_{A}}^{L}=\varphi_{F_{B}}^{L}$, and $\varphi_{F_{A}}^{U}=\varphi_{F_{B}}^{U}$.

Definition 31: The WCSM for IVCq-ROFS is given by Eq. (28) as shown in Box XII. Eq. (28) holds the following conditions:

1. $0 \leq J_{W c}^{I V}(A, B) \leq 1$,

2. $J_{W c}^{I V}(A, B)=J_{W c}^{I V}(B, A)$,

3. $J_{W c}^{I V}(A, B)=1$, for $A=B$, i.e., $T_{A}^{L}=T_{B}^{L}, T_{A}^{U}=$ $T_{B}^{U}, F_{A}^{L}=F_{B}^{L}, F_{A}^{U}=F_{B}^{U}, \varphi_{T_{A}}^{L}=\varphi_{T_{B}}^{L}, \varphi_{T_{A}}^{U}=\varphi_{T_{B}}^{U}$, $\varphi_{F_{A}}^{L}=\varphi_{F_{B}}^{L}$, and $\varphi_{F_{A}}^{U}=\varphi_{F_{B}}^{U}$.

\subsection{The HVSMs for Cq-ROFSs}

This subsection investigates the notion of HVSM called VCSM and Hybrid Weighted VSM (HWVSM) called
Weighted VCSM (WVCSM) for Cq-ROFSs and IVCqROFSs. The properties of the proposed methods are discussed in detail.

Definition 32: The HVSM for Cq-ROFS is given by Eq. (29) as shown in Box XIII. Eq. (29) holds the following conditions:

1. $0 \leq H_{V}(A, B) \leq 1$,

2. $H_{V}(A, B)=H_{V}(B, A)$,

3. $H_{V}(A, B)=1$, for $A=B$ i.e., $T_{A}=T_{B}, \varphi_{T_{A}}=$ $\varphi_{T_{B}}, F_{A}=F_{B}$, and $\varphi_{F_{A}}=\varphi_{F_{B}}$.

Proof: The proofs of the above three points are shown as follows:

1. The DSM and CSM for Cq-ROFS are proposed in Eqs. (19) and (21); we have $0 \leq J_{d}(A, B) \leq 1$ and $0 \leq J_{c}(A, B) \leq 1$. Then, we get $H_{V}(A, B)=$ $\theta J_{d}(A, B)+(1-\theta) J_{c}(A, B) \leq \theta+1-\theta=1$. Further, $0 \leq J_{d}(A, B)$ and $0 \leq J_{c}(A, B)$; therefore, $H_{V}(A, B) \geq 0$ for any values of $\theta \in[0,1]$. Hence, $0 \leq H_{V}(A, B) \leq 1$ holds clearly;

$$
J_{W c}^{I V}(A, B)=\frac{1}{2} \sum_{i=1}^{n} \omega_{i}\left(\begin{array}{l}
\frac{\left(\Omega T_{A}^{q}\left(k_{i}\right) \Omega T_{B}^{q}\left(k_{i}\right)+\Omega F_{A}^{q}\left(k_{i}\right) \Omega F_{B}^{q}\left(k_{i}\right)\right)}{\sqrt{\left(\left(\Omega T_{A}^{q}\left(k_{i}\right)\right)^{2}+\left(\Omega F_{A}^{q}\left(k_{i}\right)\right)^{2}\right)} \cdot \sqrt{\left(\left(\Omega T_{B}^{q}\left(k_{i}\right)\right)^{2}+\left(\Omega F_{B}^{q}\left(k_{i}\right)\right)^{2}\right)}} \\
+\frac{\left(\Omega \varphi_{T_{A}}^{q}\left(k_{i}\right) \Omega \varphi_{T_{B}}^{q}\left(k_{i}\right)+\Omega \varphi_{F_{A}}^{q}\left(k_{i}\right) \Omega \varphi_{F_{B}}^{q}\left(k_{i}\right)\right)}{\sqrt{\left(\left(\Omega \varphi_{T_{A}}^{q}\left(k_{i}\right)\right)^{2}+\left(\Omega \varphi_{F_{A}}^{q}\left(k_{i}\right)\right)^{2}\right)} \cdot \sqrt{\left(\left(\Omega \varphi_{T_{B}}^{q}\left(k_{i}\right)\right)^{2}+\left(\Omega \varphi_{F_{B}}^{q}\left(k_{i}\right)\right)^{2}\right)}}
\end{array}\right) .
$$




$$
H_{V}(A, B)=\frac{1}{n}\left(\begin{array}{c}
\frac{\theta}{2} \sum_{i=1}^{n}\left(\begin{array}{c}
\frac{2\left(T_{A}^{q}\left(k_{i}\right) T_{B}^{q}\left(k_{i}\right)+F_{A}^{q}\left(k_{i}\right) F_{B}^{q}\left(k_{i}\right)\right)}{\left(\left(T_{A}^{2 q}\left(k_{i}\right)+F_{A}^{2 q}\left(k_{i}\right)\right)+\left(\left(T_{B}^{2 q}\left(k_{i}\right)+F_{B}^{2 q}\left(k_{i}\right)\right)\right.\right.} \\
+\frac{2\left(\varphi_{T_{A}}^{q}\left(k_{i}\right) \varphi_{T_{B}}^{q}\left(k_{i}\right)+\varphi_{F_{A}}^{q}\left(k_{i}\right) \varphi_{F_{B}}^{q}\left(k_{i}\right)\right)}{\left(\varphi_{T_{A}}^{2 q}\left(k_{i}\right)+\varphi_{F_{A}}^{2 q}\left(k_{i}\right)\right)+\left(\varphi_{T_{B}}^{2 q}\left(k_{i}\right)+\varphi_{F_{B}}^{2 q}\left(k_{i}\right)\right)}
\end{array}\right) \\
+\frac{(1-\theta)}{2} \sum_{i=1}^{n}\left(\begin{array}{c}
\frac{\left(T_{A}^{q}\left(k_{i}\right) T_{B}^{q}\left(k_{i}\right)+F_{A}^{q}\left(k_{i}\right) F_{B}^{q}\left(k_{i}\right)\right)}{\sqrt{\left(\left(T_{A}^{2 q}\left(k_{i}\right)+F_{A}^{2 q}\left(k_{i}\right)\right)\right.} \cdot \sqrt{\left(\left(T_{B}^{2 q}\left(k_{i}\right)+F_{B}^{2 q}\left(k_{i}\right)\right)\right.}} \\
+\frac{\left(\varphi_{T_{A}}^{q}\left(k_{i}\right) \varphi_{T_{B}}^{q}\left(k_{i}\right)+\varphi_{F_{A}}^{q}\left(k_{i}\right) \varphi_{F_{B}}^{q}\left(k_{i}\right)\right)}{\sqrt{\left(\varphi_{T_{A}}^{2 q}\left(k_{i}\right)+\varphi_{F_{A}}^{2 q}\left(k_{i}\right)\right)} \cdot \sqrt{\left(\varphi_{T_{B}}^{2 q}\left(k_{i}\right)+\varphi_{F_{B}}^{2 q}\left(k_{i}\right)\right)}}
\end{array}\right)
\end{array}\right) .
$$

Box XIII

$$
H_{W V}(A, B)=\left(\begin{array}{c}
\frac{\theta}{2} \sum_{i=1}^{n} \omega_{i}\left(\begin{array}{c}
\frac{2\left(T_{A}^{q}\left(k_{i}\right) T_{B}^{q}\left(k_{i}\right)+F_{A}^{q}\left(k_{i}\right) F_{B}^{q}\left(k_{i}\right)\right)}{\left(\left(T_{A}^{2 q}\left(k_{i}\right)+F_{A}^{2 q}\left(k_{i}\right)\right)+\left(\left(T_{B}^{2 q}\left(k_{i}\right)+F_{B}^{2 q}\left(k_{i}\right)\right)\right.\right.} \\
+\frac{2\left(\varphi_{T_{A}}^{q}\left(k_{i}\right) \varphi_{T_{B}}^{q}\left(k_{i}\right)+\varphi_{F_{A}}^{q}\left(k_{i}\right) \varphi_{F_{B}}^{q}\left(k_{i}\right)\right)}{\left(\varphi_{T_{A}}^{2 q}\left(k_{i}\right)+\varphi_{F_{A}}^{2 q}\left(k_{i}\right)\right)+\left(\varphi_{T_{B}}^{2 q}\left(k_{i}\right)+\varphi_{F_{B}}^{2 q}\left(k_{i}\right)\right)}
\end{array}\right) \\
+\frac{(1-\theta)}{2} \sum_{i=1}^{n} \omega_{i}\left(\begin{array}{c}
\frac{\left(T_{A}^{q}\left(k_{i}\right) T_{B}^{q}\left(k_{i}\right)+F_{A}^{q}\left(k_{i}\right) F_{B}^{q}\left(k_{i}\right)\right)}{\sqrt{\left(\left(T_{A}^{2 q}\left(k_{i}\right)+F_{A}^{2 q}\left(k_{i}\right)\right)\right.} \cdot \sqrt{\left(\left(T_{B}^{2 q}\left(k_{i}\right)+F_{B}^{2 q}\left(k_{i}\right)\right)\right.}} \\
+\frac{\left(\varphi_{T_{A}}^{q}\left(k_{i}\right) \varphi_{T_{B}}^{q}\left(k_{i}\right)+\varphi_{F_{A}}^{q}\left(k_{i}\right) \varphi_{F_{B}}^{q}\left(k_{i}\right)\right)}{\sqrt{\left(\varphi_{T_{A}}^{2 q}\left(k_{i}\right)+\varphi_{F_{A}}^{2 q}\left(k_{i}\right)\right)} \cdot \sqrt{\left(\varphi_{T_{B}}^{2 q}\left(k_{i}\right)+\varphi_{F_{B}}^{2 q}\left(k_{i}\right)\right)}}
\end{array}\right)
\end{array}\right) .
$$

\section{Box XIV}

2. $H_{V}(A, B)=H_{V}(B, A)$ holds obviously;

3. If we consider $T_{A}=T_{B}, \varphi_{T_{A}}=\varphi_{T_{B}}, F_{A}=F_{B}$, and $\varphi_{F_{A}}=\varphi_{F_{B}}$, then the values of $J_{d}(A, B)=1$ and $J_{c}(A, B)=1$ are achieved; therefore, $H_{V}(A, B)=1$ is obtained, as well.

Definition 33: The HWVSM for Cq-ROFS is given by Eq. (30) as shown in Box XIV. Eq. (30) holds the following conditions:

1. $0 \leq H_{W V}(A, B) \leq 1$,

2. $H_{W V}(A, B)=H_{W V}(B, A)$,

3. $H_{W V}(A, B)=1$, for $A=B$, i.e., $T_{A}=T_{B}, \varphi_{T_{A}}=$ $\varphi_{T_{B}}, F_{A}=F_{B}$, and $\varphi_{F_{A}}=\varphi_{F_{B}}$.

Definition 34: The HVSM for IVCq-ROFS is given by Eq. (31) as shown in Box XV. Eq. (31) holds the following conditions:

1. $0 \leq H_{V}^{I V}(A, B) \leq 1$,
2. $H_{V}^{I V}(A, B)=H_{V}^{I V}(B, A)$,

3. $H_{V}^{I V}(A, B)=1$, for $A=B$ i.e., $T_{A}^{L}=T_{B}^{L}, T_{A}^{U}=$ $T_{B}^{U}, F_{A}^{L}=F_{B}^{L}, F_{A}^{U}=F_{B}^{U}, \varphi_{T_{A}}^{L}=\varphi_{T_{B}}^{L}, \varphi_{T_{A}}^{U}=\varphi_{T_{B}}^{U}$, $\varphi_{F_{A}}^{L}=\varphi_{F_{B}}^{L}$, and $\varphi_{F_{A}}^{U}=\varphi_{F_{B}}^{U}$.

Proof: The proofs of the above three points are shown as follows:

1. The DSM and CSM for IVCq-ROFS are proposed in Eqs. (25) and (27); we have $0 \leq J_{d}^{I V}(A, B) \leq 1$ and $0 \leq J_{c}^{I V}(A, B) \leq 1$ and then, get $H_{V}^{I V}(A, B)=$ $\theta J_{d}^{I V}(\bar{A}, B)+(1-\bar{\theta}) J_{c}^{I V}(A, B) \leq \theta+1-\theta=1$. Further, $0 \leq J_{d}^{I V}(A, B)$ and $0 \leq J_{c}^{I V}(A, B)$; thus, $H_{V}^{I V}(A, B) \geq 0$ for any values of $\theta \in[0,1]$. Hence, $0 \leq H_{V}^{I V}(A, B) \leq 1$ holds clearly;

2. $H_{V}^{I V}(A, B)=H_{V}^{I V}(B, A)$ holds clearly;

3. If we consider $T_{A}^{L}=T_{B}^{L}, T_{A}^{U}=T_{B}^{U}, F_{A}^{L}=F_{B}^{L}$, $F_{A}^{U}=F_{B}^{U}, \varphi_{T_{A}}^{L}=\varphi_{T_{B}}^{L}, \varphi_{T_{A}}^{U}=\varphi_{T_{B}}^{U}, \varphi_{F_{A}}^{L}=\varphi_{F_{B}}^{L}$, and $\varphi_{F_{A}}^{U}=\varphi_{F_{B}}^{U}$, then the values of $J_{d}^{I V}(A, B)=$ 


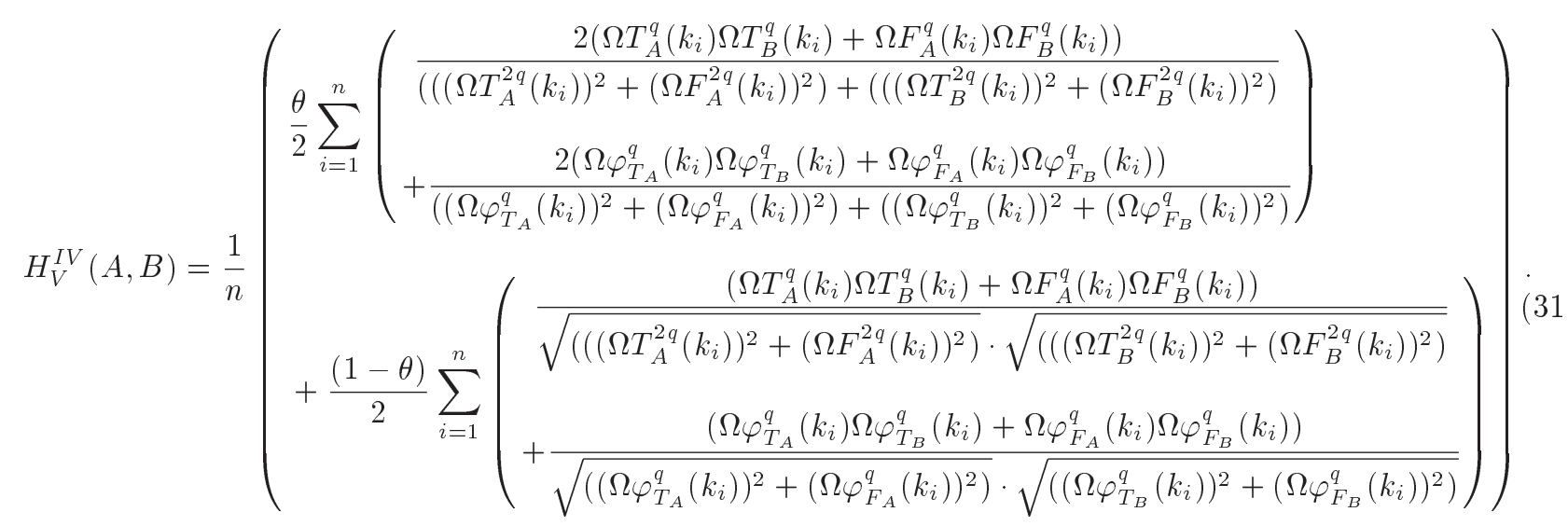

Box XV

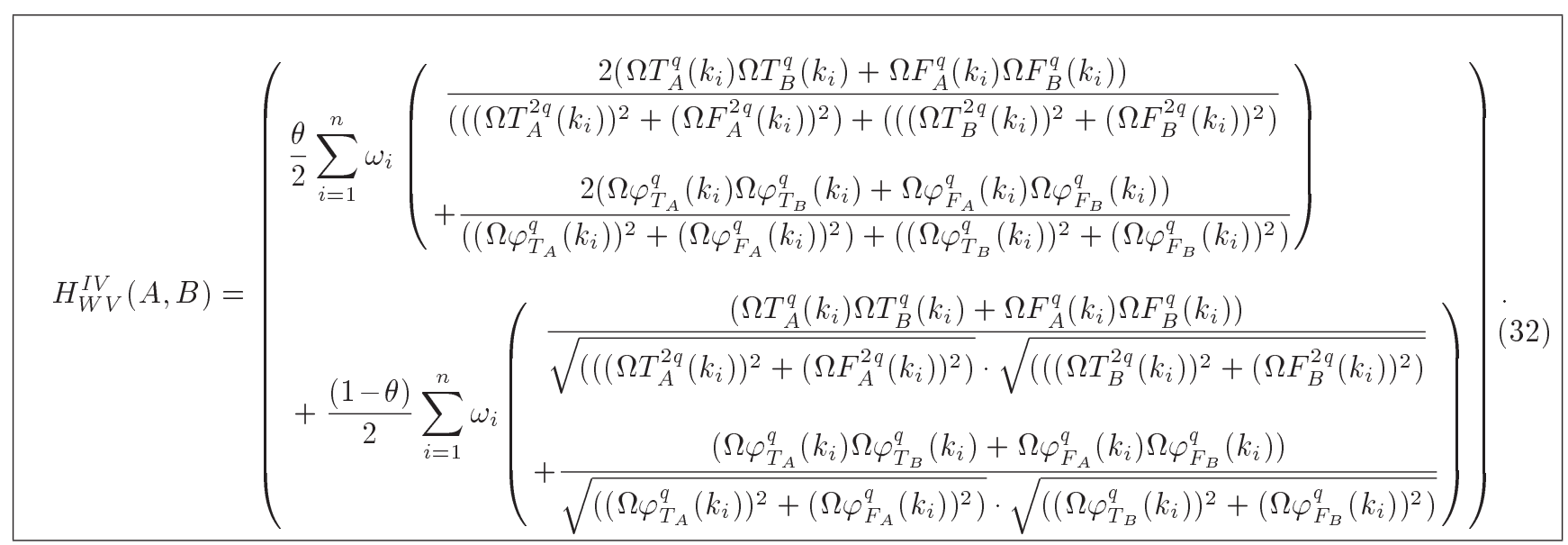

Box XVI

1 and $J_{c}^{I V}(A, B)=1$ are achieved and therefore, $H_{V}^{I V}(A, B)=1$ is obtained, as well.

Therefore, the proofs are completed.

Definition 35: The HWVSM for IVCq-ROFS is given by Eq. (32) as shown in Box XVI. Eq. (32) holds the following conditions:

1. $0 \leq H_{W V}^{I V}(A, B) \leq 1$,

2. $H_{W V}^{I V}(A, B)=H_{W V}^{I V}(B, A)$,

3. $H_{W V}^{I V}(A, B)=1$, for $A=B$ i.e., $T_{A}^{L}=T_{B}^{L}, T_{A}^{U}=$ $T_{B}^{U}, F_{A}^{L}=F_{B}^{L}, F_{A}^{U}=F_{B}^{U}, \varphi_{T_{A}}^{L}=\varphi_{T_{B}}^{L}, \varphi_{T_{A}}^{U}=\varphi_{T_{B}}^{U}$, $\varphi_{F_{A}}^{L}=\varphi_{F_{B}}^{L}$, and $\varphi_{F_{A}}^{U}=\varphi_{F_{B}}^{U}$,

where $\omega=\left\{\omega_{1}, \omega_{2}, \cdots, \omega_{n}\right\}^{T}, \omega_{i} \in[0,1], i=$ $1,2, \cdots, n$ with a condition that $\sum_{i=1}^{n} \omega_{i}=1$ represent the weighted vectors. If we consider $\omega_{i}=$ $\left(\frac{1}{n}, \frac{1}{n}, \cdots, \frac{1}{n}\right)^{T}$, then Eqs. (18), (20), and (22) are converted into Eqs. (17), (19), and (21), respectively.
Similarly, if we consider $\omega_{i}=\left(\frac{1}{n}, \frac{1}{n}, \cdots, \frac{1}{n}\right)^{T}$, then Eqs. (24), (26), and (28) are converted into Eqs. (23), (25), Eqs. (23), (25), and (27), respectively. Further, if we consider $\omega_{i}=\left(\frac{1}{n}, \frac{1}{n}, \cdots, \frac{1}{n}\right)^{T}$; then, Eqs. (30) and (32) are converted into Eqs. (29) and (31), respectively.

\section{Applications based on VSMs for Cq-ROFNs}

This section gives two applications such as medical diagnosis and pattern recognition.

\subsection{An application for medical diagnosis}

We diagnose the symptoms of diseases in a patient to determine a disease, and the corresponding steps are shown as follows.

1. Construct the matrix for symptoms $k_{j}(j=$ $1,2, \cdots, n)$ of diseases $B_{j}(j=1,2,3,4)$;

2. Give the symptoms of the patient $P$; 
Table 1. Representation of all diagnoses.

\begin{tabular}{lcccc}
\hline Symbol representations & $B_{1}$ & $B_{2}$ & $B_{3}$ & $B_{4}$ \\
Diseases & Viral fever & Malaria & Typhoid & Stomach problem \\
\hline
\end{tabular}

Table 2. Representation of all symptoms.

\begin{tabular}{lcccc}
\hline Symbol representations & $k_{1}$ & $k_{2}$ & $k_{3}$ & $k_{4}$ \\
Symptoms & Temperature & Headache & Stomach pain & Cough \\
\hline
\end{tabular}

Table 3. Decision matrix of Cq-ROFNs.

\begin{tabular}{ccccc}
\hline Values & $\boldsymbol{B}_{\mathbf{1}}$ & $\boldsymbol{B}_{\mathbf{2}}$ & $\boldsymbol{B}_{\mathbf{3}}$ & $\boldsymbol{B}_{\mathbf{4}}$ \\
\hline $\boldsymbol{k}_{\mathbf{1}}$ & $\left(0.8 e^{i 2 \pi(0.7)}, 0.1 e^{i 2 \pi(0.2)}\right)$ & $\left(0.6 e^{i 2 \pi(0.4)}, 0.1 e^{i 2 \pi(0.5)}\right)$ & $\left(0.3 e^{i 2 \pi(0.8)}, 0.3 e^{i 2 \pi(0.1)}\right)$ & $\left(0.5 e^{i 2 \pi(0.3)}, 0.4 e^{i 2 \pi(0.6)}\right)$ \\
$\boldsymbol{k}_{\mathbf{2}}$ & $\left(0.9 e^{i 2 \pi(0.6)}, 0.1 e^{i 2 \pi(0.2)}\right)$ & $\left(0.4 e^{i 2 \pi(0.9)}, 0.5 e^{i 2 \pi(0.1)}\right)$ & $\left(0.8 e^{i 2 \pi(0.3)}, 0.1 e^{i 2 \pi(0.6)}\right)$ & $\left(0.3 e^{i 2 \pi(0.1)}, 0.6 e^{i 2 \pi(0.3)}\right)$ \\
$\boldsymbol{k}_{\mathbf{3}}$ & $\left(0.7 e^{i 2 \pi(0.8)}, 0.2 e^{i 2 \pi(0.1)}\right)$ & $\left(0.5 e^{i 2 \pi(0.5)}, 0.3 e^{i 2 \pi(0.3)}\right)$ & $\left(0.7 e^{i 2 \pi(0.6)}, 0.2 e^{i 2 \pi(0.2)}\right)$ & $\left(0.8 e^{i 2 \pi(0.3)}, 0.1 e^{i 2 \pi(0.5)}\right)$ \\
$\boldsymbol{k}_{\mathbf{4}}$ & $\left(0.8 e^{i 2 \pi(0.7)}, 0.2 e^{i 2 \pi(0.1)}\right)$ & $\left(0.4 e^{i 2 \pi(0.9)}, 0.5 e^{i 2 \pi(0.1)}\right)$ & $\left(0.2 e^{i 2 \pi(0.7)}, 0.8 e^{i 2 \pi(0.2)}\right)$ & $\left(0.1 e^{i 2 \pi(0.3)}, 0.6 e^{i 2 \pi(0.5)}\right)$ \\
\hline
\end{tabular}

Table 4. Patients' information.

\begin{tabular}{ccccc}
\hline Symbols & $\boldsymbol{k}_{\mathbf{1}}$ & $\boldsymbol{k}_{\mathbf{2}}$ & $\boldsymbol{k}_{\mathbf{3}}$ & $\boldsymbol{k}_{\mathbf{4}}$ \\
\hline $\boldsymbol{P}$ & $\left(0.8 e^{i 2 \pi(0.6)}, 0.1 e^{i 2 \pi(0.2)}\right)$ & $\left(0.9 e^{i 2 \pi(0.7)}, 0.1 e^{i 2 \pi(0.2)}\right)$ & $\left(0.7 e^{i 2 \pi(0.8)}, 0.2 e^{i 2 \pi(0.1)}\right)$ & $\left(0.6 e^{i 2 \pi(0.5)}, 0.2 e^{i 2 \pi(0.4)}\right)$ \\
\hline
\end{tabular}

3. Using Eq. (30) to calculate the similarity measures of patient $P$ with the diseases $B_{j}(j=1,2,3,4)$;

4. Rank the similarity measures with all diseases $B_{j}$ $(j=1,2,3,4)$;

5. Determine the disease of the patient;

6. End.

Based on the above-mentioned steps, an example is used to express the reliability and effectiveness of the proposed approaches, taken from [23].

Numerical Example 1: We consider a set of all diseases shown in Table 1 . Next, we consider a set of all symptoms listed in Table 2 .

The steps for solving this problem are as follows:

1. Construct a matrix for symptoms $k_{j}(j=1,2, \cdots$, $n$ ) of diseases $B_{j}(j=1,2,3,4)$ as shown in Table 3 (suppose the weighted vector of symptoms $\omega=$ $\{0.3,0.2,0.1,0.4\}^{T}$ and $\left.q=1\right)$.

2. Determine the symptoms of the patient $P$ in terms of Cq-ROFSs in Table 4.

3. Employ Eq. (30) to calculate the VSMs of the patient $P$ with the all diseases and get:

$$
\begin{array}{ll}
H_{W V}\left(B_{1}, P\right)=0.96 ; & H_{W V}\left(B_{2}, P\right)=0.85 ; \\
H_{W V}\left(B_{3}, P\right)=0.79 ; & H_{W V}\left(B_{4}, P\right)=0.68 .
\end{array}
$$

4. Rank the similarity measures with all diseases and obtain:

$$
B_{1} \geq B_{2} \geq B_{3} \geq B_{4}
$$

5. The disease of the patient is $B_{1}$, which is a viral fever.

6. End.

The comparison between the proposed and existing methods with respect to this example is shown in Table 5. Further, we can give a graphical interpretation of Table 5, which is shown in Figure 2.

From Table 5, we know that all methods get the same disease for this patient, i.e., it is $B_{1}$.

Next, we will give another example for pattern recognition.

\subsection{Another application for pattern recognition}

The steps for solving the application of pattern recognition are shown as follows:

1. Construct the decision matrix for known patterns $B_{i}(i=1,2, \cdots, m)$ and criteria $(j=1,2, \cdots, n)$;

2. Get the criteria values associated with the unknown pattern $P$;

3. Use Eq. (32) to examine the similarity measures between known patterns $B_{i}(j=1,2,3,4)$ with respect to unknown pattern $P$; 
Table 5. Comparison between the proposed method and existing methods.

\begin{tabular}{|c|c|c|}
\hline Methods & Score functions & Ranking \\
\hline CIFS proposed by Rani and Garg [20] & $\begin{array}{l}H_{W V}\left(B_{1}, P\right)=0.96 ; H_{W V}\left(B_{2}, P\right)=0.85 \\
H_{W V}\left(B_{3}, P\right)=0.79 ; H_{W V}\left(B_{4}, P\right)=0.68\end{array}$ & $B_{1}>B_{2}>B_{3}>B_{4}$ \\
\hline CIFS proposed by Garg and Rani [23] & $\begin{array}{l}H_{W V}\left(B_{1}, P\right)=0.02 ; H_{W V}\left(B_{2}, P\right)=0.006 \\
H_{W V}\left(B_{3}, P\right)=0.008 ; H_{W V}\left(B_{4}, P\right)=0.01\end{array}$ & $B_{1}>B_{4}>B_{3}>B_{2}$ \\
\hline CPFS proposed by Ullah et al. [26] & $\begin{array}{l}H_{W V}\left(B_{1}, P\right)=0.29 ; H_{W V}\left(B_{2}, P\right)=0.24 \\
H_{W V}\left(B_{3}, P\right)=0.26 ; H_{W V}\left(B_{4}, P\right)=0.28\end{array}$ & $B_{1}>B_{4}>B_{3}>B_{2}$ \\
\hline Proposed methods in this paper for $q=1$ & $\begin{array}{l}H_{W V}\left(B_{1}, P\right)=0.96 ; H_{W V}\left(B_{2}, P\right)=0.85 \\
H_{W V}\left(B_{3}, P\right)=0.79 ; H_{W V}\left(B_{4}, P\right)=0.68\end{array}$ & $B_{1}>B_{2}>B_{3}>B_{4}$ \\
\hline Proposed methods in this paper for $q=2$ & $\begin{array}{l}H_{W V}\left(B_{1}, P\right)=0.23 ; H_{W V}\left(B_{2}, P\right)=0.15 \\
H_{W V}\left(B_{3}, P\right)=0.14 ; H_{W V}\left(B_{4}, P\right)=0.08\end{array}$ & $B_{1}>B_{2}>B_{3}>B_{4}$ \\
\hline Proposed methods in this paper for $q=5$ & $\begin{aligned} H_{W V}\left(B_{1}, P\right) & =0.019 ; H_{W V}\left(B_{2}, P\right)=0.006 \\
H_{W V}\left(B_{3}, P\right) & =0.008 ; H_{W V}\left(B_{4}, P\right)=0.001\end{aligned}$ & $B_{1}>B_{3}>B_{2}>B_{4}$ \\
\hline
\end{tabular}

Table 6. Representation of all alternatives.

\begin{tabular}{lcccc}
\hline Symbol representations & $B_{1}$ & $B_{2}$ & $B_{3}$ & $B_{4}$ \\
Alternatives & Car company & Food company & Computer company & Arms company \\
\hline
\end{tabular}

Table 7. Representation of all criteria.

\begin{tabular}{lcccc}
\hline Symbol representations & $k_{1}$ & $k_{2}$ & $k_{3}$ & $k_{4}$ \\
Criteria & Risk analysis & Growth analysis & Social impact & Political impact \\
\hline
\end{tabular}

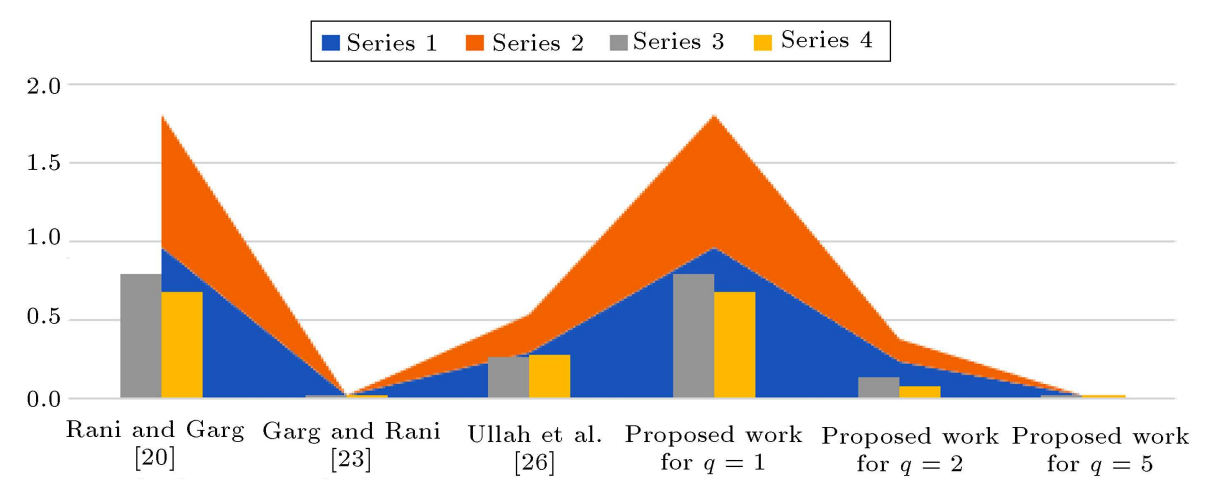

Figure 2. Graphical interpretation of the proposed work with existing works in Table 5.

4. Rank the similarity measures $B_{i}(j=1,2,3,4)$;

5. Choose the most desirable pattern $B_{i}(j=1,2,3,4)$ to be classified with pattern $P$;

6. End.

Numerical Example 2: Here we give detailed information. Firstly, a set of all possible alternatives is considered in Table 6 . Next, a set of all the criteria listed in Table 7 is considered. The steps of this decision are shown below:

1. Construct the decision matrix for the alternatives with respect to all the criteria, which is expressed by IvCq-ROFNs shown in Table 8 (the weighted vector of criteria is $\left.\omega=\{0.3,0.2,0.1,0.4\}^{T}\right)$;

2. Determine the information on the criteria associated with alternative $P$, as given in Table 9; 
Table 8. Decision matrix of IVCq-ROFNs in Example 2.

\begin{tabular}{ccccc}
\hline Values & $\boldsymbol{B}_{\mathbf{1}}$ & $\boldsymbol{B}_{\mathbf{2}}$ & $\boldsymbol{B}_{\mathbf{3}}$ & $\boldsymbol{B}_{\mathbf{4}}$ \\
\hline $\boldsymbol{k}_{\mathbf{1}}$ & $\left(\begin{array}{c}{[0.7,0.9] e^{i 2 \pi[0.5,0.9]}} \\
{[0.1,0.5] e^{i 2 \pi[0.2,0.6]}}\end{array}\right)$ & $\left(\begin{array}{c}{[0.5,0.7] e^{i 2 \pi[0.3,0.5]}} \\
{[0.2,0.4] e^{i 2 \pi[0.5,0.9]}}\end{array}\right)$ & $\left(\begin{array}{c}{[0.1,0.5] e^{i 2 \pi[0.7,0.9]}} \\
{[0.4,0.6] e^{i 2 \pi[0.2,0.4]}}\end{array}\right)$ & $\left(\begin{array}{c}{[0.4,0.6] e^{i 2 \pi[0.2,0.4]},} \\
{[0.3,0.9] e^{i 2 \pi[0.4,0.8]}}\end{array}\right)$ \\
$\boldsymbol{k}_{\mathbf{2}}$ & $\left(\begin{array}{c}{[0.89,0.91] e^{i 2 \pi[0.5,0.7]},} \\
{[0.1,0.5] e^{i 2 \pi[0.2,0.6]}}\end{array}\right)$ & $\left(\begin{array}{c}{[0.3,0.5] e^{i 2 \pi[0.89,0.91]},} \\
{[0.5,0.9] e^{i 2 \pi[0.2,0.4]}}\end{array}\right)$ & $\left(\begin{array}{l}{[0.7,0.9] e^{i 2 \pi[0.2,0.4]},} \\
{[0.2,0.4] e^{i 2 \pi[0.7,0.9]}}\end{array}\right)$ & $\left(\begin{array}{c}{[0.1,0.5] e^{i 2 \pi[0.01,0.19]},} \\
{[0.7,0.9] e^{i 2 \pi[0.2,0.4]}}\end{array}\right)$ \\
$\boldsymbol{k}_{\mathbf{3}}$ & $\left(\begin{array}{c}{[0.5,0.9] e^{i 2 \pi[0.7,0.9]},} \\
{[0.2,0.6] e^{i 2 \pi[0.1,0.5]}}\end{array}\right)$ & $\left(\begin{array}{c}{[0.4,0.6] e^{i 2 \pi[0.4,0.6]},} \\
{[0.4,0.6] e^{i 2 \pi[0.4,0.6]}}\end{array}\right)$ & $\left(\begin{array}{c}{[0.6,0.8] e^{i 2 \pi[0.5,0.7]},} \\
{[0.39,0.41] e^{i 2 \pi[0.39,0.41]}}\end{array}\right)$ & $\left(\begin{array}{c}{[0.7,0.9] e^{i 2 \pi[0.2,0.4]},} \\
{[0.2,0.4] e^{i 2 \pi[0.4,0.6])}}\end{array}\right)$ \\
$\boldsymbol{k}_{\mathbf{4}}$ & $\left(\begin{array}{c}{[0.7,0.9] e^{i 2 \pi[0.5,0.9]},} \\
{[0.2,0.6] e^{i 2 \pi[0.1,0.5]}}\end{array}\right)$ & $\left(\begin{array}{c}{[0.3,0.5] e^{i 2 \pi[0.89,0.91]},} \\
{[0.5,0.9] e^{i 2 \pi[0.1,0.5]}}\end{array}\right)$ & $\left(\begin{array}{c}{[0.39,0.41] e^{i 2 \pi[0.5,0.9]}} \\
{[0.7,0.9] e^{i 2 \pi[0.39,0.41]}}\end{array}\right)$ & $\left(\begin{array}{c}\left([0.01,0.19] e^{i 2 \pi[0.2,0.4]},\right. \\
{[0.5,0.7] e^{i 2 \pi[0.4,0.6]}}\end{array}\right)$ \\
\hline
\end{tabular}

Table 9. Information for alternative $P$.

\begin{tabular}{|c|c|c|c|c|}
\hline Symbols & $k_{1}$ & $k_{2}$ & $k_{3}$ & $k_{4}$ \\
\hline$P$ & $\left(\begin{array}{c}{[0.7,0.9] e^{i 2 \pi[0.5,0.7]},} \\
{[0.2,0.4] e^{i 2 \pi[0.3,0.5]}}\end{array}\right)$ & $\left(\begin{array}{c}{[0.89,0.91] e^{i 2 \pi[0.6,0.8]},} \\
{[0.2,0.4] e^{i 2 \pi[0.3,0.5]}}\end{array}\right)$ & $\left(\begin{array}{c}{[0.6,0.8] e^{i 2 \pi[0.7,0.9]},} \\
{[0.1,0.7] e^{i 2 \pi[0.2,0.4]}}\end{array}\right)$ & $\left(\begin{array}{c}{[0.4,0.8] e^{i 2 \pi[0.4,0.6]},} \\
{[0.3,0.5] e^{i 2 \pi[0.5,0.7]}}\end{array}\right)$ \\
\hline
\end{tabular}

Table 10. Decision matrix of Cq-ROFNs in Example 3.

\begin{tabular}{ccccc}
\hline Values & $\boldsymbol{B}_{\mathbf{1}}$ & $\boldsymbol{B}_{\mathbf{2}}$ & $\boldsymbol{B}_{\mathbf{3}}$ & $\boldsymbol{B}_{\mathbf{4}}$ \\
\hline $\boldsymbol{k}_{\boldsymbol{1}}$ & $\left(0.8 e^{i 2 \pi(0.7)}, 0.3 e^{i 2 \pi(0.4)}\right)$ & $\left(0.6 e^{i 2 \pi(0.4)}, 0.3 e^{i 2 \pi(0.7)}\right)$ & $\left(0.3 e^{i 2 \pi(0.8)}, 0.5 e^{i 2 \pi(0.3)}\right)$ & $\left(0.5 e^{i 2 \pi(0.3)}, 0.6 e^{i 2 \pi(0.8)}\right)$ \\
$\boldsymbol{k}_{\boldsymbol{2}}$ & $\left(0.9 e^{i 2 \pi(0.6)}, 0.3 e^{i 2 \pi(0.4)}\right)$ & $\left(0.4 e^{i 2 \pi(0.9)}, 0.7 e^{i 2 \pi(0.3)}\right)$ & $\left(0.8 e^{i 2 \pi(0.3)}, 0.3 e^{i 2 \pi(0.8)}\right)$ & $\left(0.3 e^{i 2 \pi(0.1)}, 0.8 e^{i 2 \pi(0.5)}\right)$ \\
$\boldsymbol{k}_{\boldsymbol{3}}$ & $\left(0.7 e^{i 2 \pi(0.8)}, 0.4 e^{i 2 \pi(0.3)}\right)$ & $\left(0.5 e^{i 2 \pi(0.5)}, 0.5 e^{i 2 \pi(0.5)}\right)$ & $\left(0.7 e^{i 2 \pi(0.6)}, 0.4 e^{i 2 \pi(0.4)}\right)$ & $\left(0.8 e^{i 2 \pi(0.3)}, 0.3 e^{i 2 \pi(0.7)}\right)$ \\
$\boldsymbol{k}_{\boldsymbol{4}}$ & $\left(0.8 e^{i 2 \pi(0.7)}, 0.4 e^{i 2 \pi(0.3)}\right)$ & $\left(0.4 e^{i 2 \pi(0.9)}, 0.7 e^{i 2 \pi(0.3)}\right)$ & $\left(0.4 e^{i 2 \pi(0.7)}, 0.8 e^{i 2 \pi(0.4)}\right)$ & $\left(0.1 e^{i 2 \pi(0.3)}, 0.8 e^{i 2 \pi(0.7)}\right)$ \\
\hline
\end{tabular}

Table 11. Decision matrix for unknown values.

\begin{tabular}{|c|c|c|c|c|}
\hline Symbols & $k_{1}$ & $k_{2}$ & $k_{3}$ & $k_{4}$ \\
\hline $\boldsymbol{P}$ & $\left(0.8 e^{i 2 \pi(0.6)}, 0.3 e^{i 2 \pi(0.4)}\right)$ & $\left(0.9 e^{i 2 \pi(0.7)}, 0.3 e^{i 2 \pi(0.4)}\right)$ & $\left(0.7 e^{i 2 \pi(0.8)}, 0.4 e^{i 2 \pi(0.3)}\right)$ & $\left(0.6 e^{i 2 \pi(0.5)}, 0.4 e^{i 2 \pi(0.6)}\right)$ \\
\hline
\end{tabular}

3. Use Eq. (32) to calculate the VSMs and get:

$$
\begin{array}{ll}
H_{W V}^{I V}\left(B_{i}, P\right)=0.075 ; & H_{W V}^{I V}\left(B_{2}, P\right)=0.042 ; \\
H_{W V}^{I V}\left(B_{3}, P\right)=0.045 ; & H_{W V}^{I V}\left(B_{4}, P\right)=0.020 .
\end{array}
$$

4. Rank all IvCq-ROFNs and obtain:

$$
B_{1} \geq B_{3} \geq B_{2} \geq B_{4}
$$

5. Thus, $B_{1}$ is the best alternative, which is car company;

6. End.

\subsection{Comparative analysis}

This subsection utilizes a numerical example to demonstrate the reliability of the proposed method in comparison to the existing methods.
Numerical Example 3: This example is revised from Numerical Example 1.

1. The symptoms for all diseases are shown in Table 10 (suppose the weighted vectors $\omega=$ $\{0.3,0.2,0.1,0.4\}^{T}$ and $\left.q=2\right)$;

2. All symptoms of the patient $P$ in terms of $\mathrm{Cq}-$ ROFSs are given in Table 11;

3. Eq. (30) is used to calculate the VSMs of the patient $P$ with the all diseases as follows:

$$
\begin{array}{ll}
H_{W V}\left(B_{1}, P\right)=0.075 ; & H_{W V}\left(B_{2}, P\right)=0.042 ; \\
H_{W V}\left(B_{3}, P\right)=0.045 ; & H_{W V}\left(B_{4}, P\right)=0.028 .
\end{array}
$$

4. The similarity measures for all diseases are ranked below:

$$
B_{1} \geq B_{3} \geq B_{2} \geq B_{4}
$$


Table 12. Comparison between the proposed methods and existing methods for Example 3.

\begin{tabular}{ccc}
\hline \multicolumn{1}{c}{ Methods } & Score functions & Ranking \\
\hline CIFS proposed by Rani and Garg [20] & Cannot be calculated & Cannot be calculated \\
CIFS proposed by Garg and Rani [23] & Cannot be calculated & Cannot be calculated \\
& & \\
CPFS proposed by Ullah et al. [26] & $H_{W V}\left(B_{1}, P\right)=0.070 ; H_{W V}\left(B_{2}, P\right)=0.036 ;$ & $B_{1}>B_{3}>B_{2}>B_{4}$ \\
& $H_{W V}\left(B_{3}, P\right)=0.039 ; H_{W V}\left(B_{4}, P\right)=0.014$ & \\
& & \\
Proposed methods & $H_{W V}\left(B_{1}, P\right)=0.075 ; H_{W V}\left(B_{2}, P\right)=0.041 ;$ & $B_{1}>B_{3}>B_{2}>B_{4}$ \\
\hline
\end{tabular}

Table 13. Decision matrix of Cq-ROFNs in Example 4.

\begin{tabular}{ccccc}
\hline Values & $\boldsymbol{B}_{\mathbf{1}}$ & $\boldsymbol{B}_{\mathbf{2}}$ & $\boldsymbol{B}_{\mathbf{3}}$ & $\boldsymbol{B}_{\mathbf{4}}$ \\
\hline $\boldsymbol{k}_{\mathbf{1}}$ & $\left(0.8 e^{i 2 \pi(0.7)}, 0.7 e^{i 2 \pi(0.4)}\right)$ & $\left(0.6 e^{i 2 \pi(0.4)}, 0.8 e^{i 2 \pi(0.7)}\right)$ & $\left(0.89 e^{i 2 \pi(0.8)}, 0.5 e^{i 2 \pi(0.3)}\right)$ & $\left(0.86 e^{i 2 \pi(0.3)}, 0.6 e^{i 2 \pi(0.8)}\right)$ \\
$\boldsymbol{k}_{\mathbf{2}}$ & $\left(0.9 e^{i 2 \pi(0.6)}, 0.8 e^{i 2 \pi(0.4)}\right)$ & $\left(0.4 e^{i 2 \pi(0.9)}, 0.9 e^{i 2 \pi(0.3)}\right)$ & $\left(0.8 e^{i 2 \pi(0.3)}, 0.3 e^{i 2 \pi(0.8)}\right)$ & $\left(0.87 e^{i 2 \pi(0.1)}, 0.8 e^{i 2 \pi(0.5)}\right)$ \\
$\boldsymbol{k}_{\mathbf{3}}$ & $\left(0.7 e^{i 2 \pi(0.8)}, 0.6 e^{i 2 \pi(0.3)}\right)$ & $\left(0.5 e^{i 2 \pi(0.5)}, 0.9 e^{i 2 \pi(0.5)}\right)$ & $\left(0.7 e^{i 2 \pi(0.6)}, 0.4 e^{i 2 \pi(0.4)}\right)$ & $\left(0.8 e^{i 2 \pi(0.3)}, 0.3 e^{i 2 \pi(0.7)}\right)$ \\
$\boldsymbol{k}_{\mathbf{4}}$ & $\left(0.8 e^{i 2 \pi(0.7)}, 0.7 e^{i 2 \pi(0.3)}\right)$ & $\left(0.4 e^{i 2 \pi(0.9)}, 0.8 e^{i 2 \pi(0.3)}\right)$ & $\left(0.8 e^{i 2 \pi(0.7)}, 0.8 e^{i 2 \pi(0.4)}\right)$ & $\left(0.9 e^{i 2 \pi(0.3)}, 0.8 e^{i 2 \pi(0.7)}\right)$ \\
\hline
\end{tabular}

Table 14. Symptoms of the patient $P$ in Example 4.

\begin{tabular}{ccccc}
\hline Symbols & $\boldsymbol{k}_{\mathbf{1}}$ & $\boldsymbol{k}_{\mathbf{2}}$ & $\boldsymbol{k}_{\mathbf{3}}$ & $\boldsymbol{k}_{\mathbf{4}}$ \\
\hline $\boldsymbol{P}$ & $\left(0.8 e^{i 2 \pi(0.6)}, 0.8 e^{i 2 \pi(0.4)}\right)$ & $\left(0.9 e^{i 2 \pi(0.7)}, 0.8 e^{i 2 \pi(0.4)}\right)$ & $\left(0.7 e^{i 2 \pi(0.8)}, 0.89 e^{i 2 \pi(0.3)}\right)$ & $\left(0.6 e^{i 2 \pi(0.5)}, 0.88 e^{i 2 \pi(0.6)}\right)$ \\
\hline
\end{tabular}

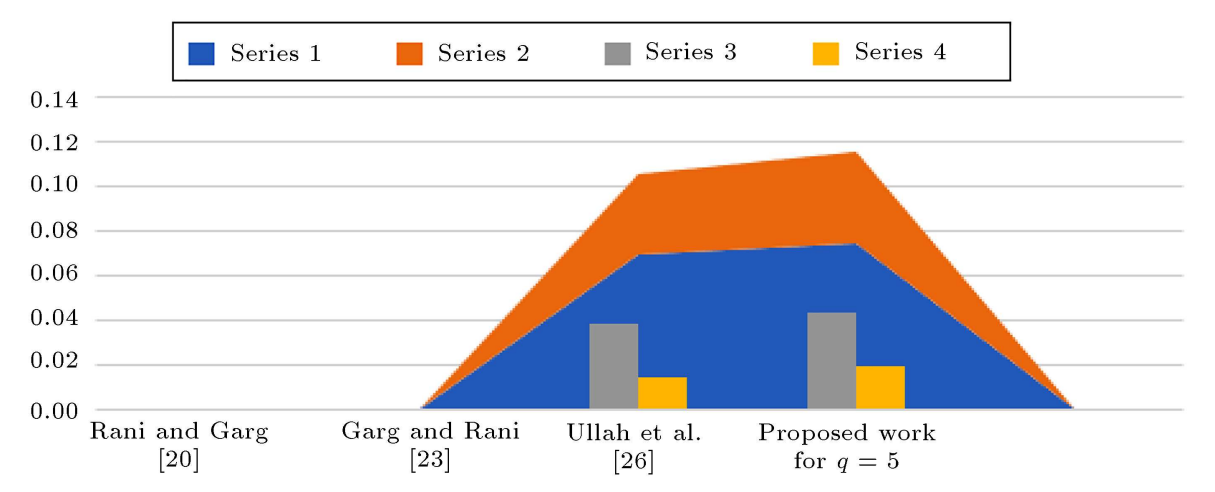

Figure 3. Comparison between the proposed methods and existing methods for Example 3.

The disease of the patient is $B_{1}$, which is a viral fever.

5. End.

The comparison between the proposed method and existing methods with respect to Example 3 is shown in Table 12. Further, a graphical interpretation of Table 12 is shown in Figure 3.

From Table 12, it is clear that our proposed method is more effective and more general to cope with uncertain and complex information. Obviously, in this example, the CIFS cannot be used to solve this decision problem; thus, the methods proposed in [20,23] are invalid. In addition, it can be seen that the CPFS can solve this example and get the same ranking result as that produced by the proposed method; hence, the validity of the proposed method is proven. In the next example, the advantage of the proposed method versus the method proposed by Ullah et al. [26] is shown further. 
Numerical Example 4: This example is the modified form of Numerical Example 1.

1. The symptoms for all diseases are shown in Table 13 (suppose the weighted vectors $\omega=$ $\{0.3,0.2,0.1,0.4\}^{T}$ and $\left.q=2\right)$;

2. All symptoms of the patient $P$ in terms of $\mathrm{Cq}^{-}$ ROFSs are denoted in Table 14;

3. Eq. (30) is employed to calculate the VSMs of the patient $P$ with the all diseases:

$$
\begin{array}{ll}
H_{W V}\left(B_{1}, P\right)=0.086 ; & H_{W V}\left(B_{2}, P\right)=0.053 ; \\
H_{W V}\left(B_{3}, P\right)=0.056 ; & H_{W V}\left(B_{4}, P\right)=0.039 .
\end{array}
$$

4. The similarity measures for all diseases are ranked below:

$$
B_{1} \geq B_{3} \geq B_{2} \geq B_{4} .
$$

Thus, the disease of the patient is $B_{1}$, which is a viral fever;

5. End.

The comparison between the proposed method and existing methods with respect to Example 4 is shown in Table 15.

From Table 15, it is clear that our proposed method is more effective and more general to cope with uncertain and complex information. Further, a graphical interpretation of Table 15 is shown in Figure 4.
Obviously, the proposed method can provide a much information expression in decision-making problems.

\subsection{Advantages of the proposed similarity measures}

The similarity measures investigated in this paper are the extension of existing ones in [54], and the proposed method is the generalization of the existing methods discussed in [54].

Remark 1: When $q=2$, Eqs. (17) and (18) are converted into JSM and WJSM of CPFSs, and when $q=1$, Eqs. (17) and (18) are converted to JSM and WJSM for CIFSs.

Remark 2: When $q=2$, Eqs. (19) and (20) are converted into DSM and WDSM of CPFSs, and when $q=1$, Eqs. (19) and (20) are converted into DSM and WDSM for CIFSs.

Remark 3: When $q=2$, Eqs. (21) and (22) are converted into CSM and WCSM of CPFSs, and when $q=1$, Eqs. (21) and (22) are converted into CSM and WCSM for CIFSs.

Remark 4: When $q=2$, Eqs. (23) and (24) are converted into JSM and WJSM of IVCPFSs, and when $q=1$, Eqs. (23) and (24) are converted into JSM and WJSM for IVCIFSs.

Table 15. Comparison between the proposed methods and existing methods for Example 4.

\begin{tabular}{lcc}
\hline \multicolumn{1}{c}{ Methods } & Score functions & Ranking \\
\hline CIFS proposed by Rani and Garg [20] & Cannot be calculated & Cannot be calculated \\
CIFS proposed by Garg and Rani [23] & Cannot be calculated & Cannot be calculated \\
CPFS proposed by Ullah et al. [26] & Cannot be calculated & Cannot be calculated \\
Proposed methods & $H_{W V}\left(B_{1}, P\right)=0.086 ; H_{W V}\left(B_{2}, P\right)=0.053 ;$ & $B_{1} \geq B_{3} \geq B_{2} \geq B_{4}$ \\
\hline
\end{tabular}

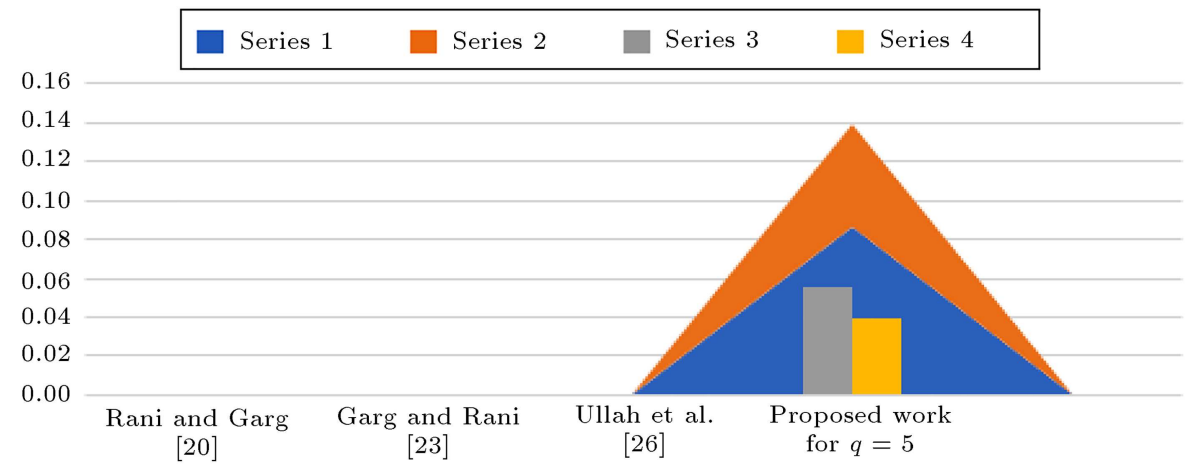

Figure 4. Comparison between the proposed methods and existing methods for Example 4. 
Remark 5: When $q=2$, Eqs. (25) and (26) are converted into DSM and WDSM of IVCPFSs, and when $q=1$, Eqs. (25) and (26) are converted into DSM and WDSM for IVCIFSs.

Remark 6: When $q=2$, Eqs. (27) and (28) are converted into CSM and WCSM of IVCPFSs, and when $q=1$, Eqs. (27) and (28) are converted into CSM and WCSM for IVCIFSs.

Remark 7: When $q=2$, Eqs. (29) and (30) are converted into HVSM and HWVSM of CPFSs, and when $q=1$, Eqs. (29) and (30) are converted into HVSM and HWVSM for CIFSs.

Remark 8: When $q=2$, Eqs. (31) and (32) are converted into HVSM and HWVSM of IVCPFSs, and when $q=1$, Eqs. (31) and (32) are converted into HVSM and HWVSM for IVCIFSs.

In a word, our proposed method is more reliable and more general than existing methods, to be described below.

Few years ago, the CIFS [15] was successfully applied in different areas; yet, it faced some real-world problems. The CIFS cannot effectively elaborate on complex kinds of information in real decision-making. In order to handle these problems, Ullah et al. [26] proposed the concept of CPFS and its constraint condition was that the sum of the square of the real membership (also for imaginary) and square of the real non-membership (also for imaginary) was limited to $[0,1]$. Obviously, the CPFS was more general than CIFS. However, the problem that remains is that when a decision-maker provides a flawed type of information, it does not satisfy the conditions of CIFS and CPFS. To deal with this constraint, the Cq-ROFSs, characterized by complex-valued membership and complex-valued non-membership, are proposed here. The advantage of the Cq-ROFS is that the sum of the q-power of the real membership (also for imaginary) and q-power of the real non-membership (also for imaginary) is bound to $[0,1]$. The Cq-ROFSs are more generalizable than CIFSs and CPFSs in dealing with uncertain and complicated information in the fuzzy set theory. For example, when a decision-maker assigns $\left(0.9 e^{i 2 \pi(0.78)}\right)$ to complex-valued membership grade and $\left(0.88 e^{i 2 \pi(0.80)}\right)$ to complex-valued non-membership grade, respectively, the validity holds only for the case of Cq-ROFS. On the other hand, all the complex intuitionistic fuzzy degrees and complex Pythagorean fuzzy degrees are part of the complex q-rung orthopair fuzzy degrees, thus demonstrating that Cq-ROFS is more powerful and more comprehensive than CIFS and CPFS.

In addition, the similarity measures for IFS, CIFS, PFS, q-ROFS, and CPFS are the special cases of the proposed similarity measures. Therefore, the proposed method is more general and more reliable in solving the real-life problem more accurately than existing methods.

\section{Conclusion}

This study introduced the background of IFSs, CIFSs, PFSs, CPFSs, and q-ROFSs in detail and explained their limitations in expressing uncertain and complex information. Further, the Cq-ROFS and IVCq-ROFS were proposed and their fundamental properties were discussed. At the same time, some numerical examples were given to explain these concepts. Obviously, the Cq-ROFS was an extension of CFS, CPFS, PFS, IFS, and FS, and it managed to express the uncertain and complicated fuzzy information better. In addition, the Cq-ROFS contains two-dimensional information in a single set whose restriction is that the sum of the q-power of the real part of the membership (also for imaginary part) and q-power of the real part of the non-membership (also for imaginary part) is less than or equal to 1 . Based on these advantages, the VSMs including Jaccard, dice, and cosine similarity measures for Cq-ROFSs and IVCq-ROFSs were investigated. Further, the HVSMs called VCSMs for CqROFSs and IVCq-ROFSs were proposed and their fundamental properties were discussed. To demonstrate the feasibility of the proposed HVSMs, the existing similarity measures based on CPFSs and CIFSs were compared with the proposed methods with respect to some numerical examples such as medical diagnosis and pattern recognition.

In the future, we will research some new similarity measures for different fuzzy sets such as similarity measures [55,56], linguistic neutrosophic set [57], probabilistic linguistic information [58], linguistic D number [59], and interval type-2 fuzzy set [60] or apply them to solve the MADM or MAGDM problems [61-64].

\section{Acknowledgment}

This paper is supported by the National Natural Science Foundation of China (No. 71771140), Project of cultural masters and "the four kinds of a batch" talents, the Special Funds of Taishan Scholars Project of Shandong Province (No. ts201511045), and Major bidding projects of National Social Science Fund of China (19ZDA080).

\section{References}

1. Atanassov, K.T. "Intuitionistic fuzzy sets", In Intuitionistic Fuzzy Sets, Physica, Heidelberg, pp. 1-137 (1999). 
2. Zadeh, L.A. "Fuzzy sets", Information and Control, 8(3), pp. 338-353 (1965).

3. Hung, W.L. and Yang, M.S. "Similarity measures of intuitionistic fuzzy sets based on Hausdorff distance", Pattern Recognition Letters, 25(14), pp. 1603-1611 (2004).

4. Xu, Z. and Xia, M. "Distance and similarity measures for hesitant fuzzy sets", Information Sciences, 181(11), pp. 2128-2138 (2011).

5. Xu, Z. and Chen, J. "An overview of distance and similarity measures of intuitionistic fuzzy sets", International Journal of Uncertainty, Fuzziness and Knowledge-Based Systems, 16(04), pp. 529-555 (2008).

6. Szmidt, E. and Kacprzyk, J. "Entropy for intuitionistic fuzzy sets", Fuzzy Sets and Systems, 118(3), pp. 467477 (2001).

7. Burillo, P. and Bustince, H. "Entropy on intuitionistic fuzzy sets and on interval-valued fuzzy sets", Fuzzy Sets and Systems, 78(3), pp. 305-316 (1996).

8. Hung, W.L. and Yang, M.S. "Fuzzy entropy on intuitionistic fuzzy sets", International Journal of Intelligent Systems, 21(4), pp. 443-451 (2006).

9. De, S.K., Biswas, R., and Roy, A. "An application of intuitionistic fuzzy sets in medical diagnosis", Fuzzy sets and Systems, 117(2), pp. 209-213 (2001).

10. Szmidt, E. and Kacprzyk, J. "A similarity measure for intuitionistic fuzzy sets and its application in supporting medical diagnostic reasoning", In International Conference on Artificial Intelligence and Soft Computing, Zakopane, Poland, pp. 388-393 (2004).

11. Szmidt, E. and Kacprzyk, J. "Intuitionistic fuzzy sets in some medical applications", In International Conference on Computational Intelligence, Dortmund, Germany, pp. 148-151 (2001).

12. Wei, G. "Some induced geometric aggregation operators with intuitionistic fuzzy information and their application to group decision making", Applied Soft Computing, 10(2), pp. 423-431 (2010).

13. Xu, Z. "Approaches to multiple attribute group decision making based on intuitionistic fuzzy power aggregation operators", Knowledge-Based Systems, 24(6), pp. 749-760 (2011).

14. Liu, P. "Some Hamacher aggregation operators based on the interval-valued intuitionistic fuzzy numbers and their application to group decision making", IEEE Transactions on Fuzzy Systems, 22(1), pp. 83-97 (2013).

15. Alkouri, A.M. and Salleh, A.R. "Complex intuitionistic fuzzy sets", In AIP Conference Proceedings, Puertollano, Spain, 1482(1), pp. 464-470 (2012).

16. Ramot, D., Milo, R., Friedman, M., et al. "Complex fuzzy sets", IEEE Transactions on Fuzzy Systems, 10(2), pp. 171-186 (2002).
17. Kumar, T. and Bajaj, R.K. "On complex intuitionistic fuzzy soft sets with distance measures and entropies", Journal of Mathematics, 2014, pp. 1-12, Article ID 972198 (2014).

18. Maji, P.K., Biswas, R., and Roy, A. "Soft set theory", Computers \& Mathematics with Applications, 45(4-5), pp. 555-562 (2003).

19. Alkouri, A.U.M. and Salleh, A.R. "Complex Atanassov's intuitionistic fuzzy relation", Abstract and Applied Analysis, 2013, pp. 1-18, Article ID 287382 (2013).

20. Rani, D. and Garg, H. "Distance measures between the complex intuitionistic fuzzy sets and their applications to the decision-making process", International Journal for Uncertainty Quantification, 7(5), pp. 423-439 (2017).

21. Rani, D. and Garg, H. "Complex intuitionistic fuzzy power aggregation operators and their applications in multicriteria decision-making", Expert Systems, 35(6), p. e12325 (2018).

22. Garg, H. and Rani, D. "Some generalized complex intuitionistic fuzzy aggregation operators and their application to multicriteria decision-making process", Arabian Journal for Science and Engineering, 44(3), pp. 2679-2698 (2019).

23. Garg, H. and Rani, D. "A robust correlation coefficient measure of complex intuitionistic fuzzy sets and their applications in decision-making", Applied Intelligence, 49(2), pp. 496-512 (2019).

24. Yager, R.R. "Pythagorean fuzzy subsets", In 2013 Joint IFSA World Congress and NAFIPS Annual Meeting (IFSA/NAFIPS), Edmonton, Canada, pp. 57-61 (2013).

25. Yager, R.R. and Abbasov, A.M. "Pythagorean membership grades, complex numbers, and decision making", International Journal of Intelligent Systems, 28(5), pp. 436-452 (2013).

26. Ullah, K., Mahmood, T., Ali, Z., et al. "On some distance measures of complex Pythagorean fuzzy sets and their applications in pattern recognition", Complex \& Intelligent Systems, 6, pp. 15-27 (2020).

27. Yager, R.R. "Generalized orthopair fuzzy sets", IEEE Transactions on Fuzzy Systems, 25(5), pp. 1222-1230 (2016).

28. Liu, Z., Liu, P., and Liang, X. "Multiple attribute decision-making method for dealing with heterogeneous relationship among attributes and unknown attribute weight information under q-rung orthopair fuzzy environment", International Journal of Intelligent Systems, 33(9), pp. 1900-1928 (2018).

29. Liu, Z., Wang, S., and Liu, P. "Multiple attribute group decision making based on q-rung orthopair fuzzy Heronian mean operators", International Journal of Intelligent Systems, 33(12), pp. 2341-2363 (2018).

30. Wang, P., Wang, J., Wei, G., et al. "Similarity measures of q-rung orthopair fuzzy sets based on cosine function and their applications", Mathematics, 7(4), p. 340 (2019). 
31. Jan, N., Mahmood, T., Zedam, L., et al. "Analysis of social networks, communication networks and shortest path problems in the environment of interval-valued qrung ortho pair fuzzy graphs", International Journal of Fuzzy Systems, 21, pp. 1687-1708 (2019).

32. Liu, P. and Liu, J. "Some q-rung orthopai fuzzy Bonferroni mean operators and their application to multi-attribute group decision making", International Journal of Intelligent Systems, 33(2), pp. 315-347 (2018).

33. Liu, P. and Wang, P. "Some q-rung orthopair fuzzy aggregation operators and their applications to multipleattribute decision making", International Journal of Intelligent Systems, 33(2), pp. 259-280 (2018).

34. Liu, P. and Wang, P. "Multiple-attribute decisionmaking based on archimedean Bonferroni operators of q-rung orthopair fuzzy numbers", IEEE Transactions on Fuzzy Systems, 27(5), pp. 834-848 (2018).

35. Peng, X., Dai, J., and Garg, H. "Exponential operation and aggregation operator for q-rung orthopair fuzzy set and their decision-making method with a new score function", International Journal of Intelligent Systems, 33(11), pp. 2255-2282 (2018).

36. Liu, P., Chen, S.M., and Wang, P. "Multiple-attribute group decision-making based on q-rung orthopair fuzzy power maclaurin symmetric mean operators", IEEE Transactions on Systems, Man, and Cybernetics: Systems, 50(10), pp. 3741-3756 (2018). DOI: 10.1109/TSMC.2018.2852948

37. Yang, W. and Pang, Y. "New q-rung orthopair fuzzy partitioned Bonferroni mean operators and their application in multiple attribute decision making", International Journal of Intelligent Systems, 34(3), pp. 439-476 (2019).

38. Li, L., Zhang, R., and Shang, X. "Some q-rung orthopair linguistic Heronian meanoperators with their application to multi-attribute group decision making", Archives of Control Sciences, 28(4), pp. 551-583 (2018).

39. Liu, P. and Liu, W. "Multiple-attribute group decisionmaking based on power Bonferroni operators of linguistic q-rung orthopair fuzzy numbers", International Journal of Intelligent Systems, 34(4), pp. 652-689 (2019).

40. Li, D.F. and Cheng, C.T. "New similarity measures of intuitionistic fuzzy sets and application to pattern recognitions", Pattern Recognition Letters, 23(1-3), pp. 221-225 (2002).

41. Mitchell, H.B. "On the Dengfeng-Chuntian similarity measure and its application to pattern recognition", Pattern Recognition Letters, 24(16), pp. 3101-3104 (2003).
42. Castellano, G., Fanelli, A.M., Mencar, C., and Torsello, M.A. "Similarity-based fuzzy clustering for user profiling", In $200^{7} \mathrm{IEEE} / W I C / A C M$ International Conferences on Web Intelligence and Intelligent Agent Technology-Workshops, California, United States, pp. 75-78 (2007).

43. Son, L.H. "Generalized picture distance measure and applications to picture fuzzy clustering", Applied Soft Computing, 46, pp. 284-295 (2016).

44. He, Q. and Wu, C. "Membership evaluation and feature selection for fuzzy support vector machine based on fuzzy rough sets", Soft Computing, 15(6), pp. 1105-1114 (2011).

45. Liu, P. and Jin, F. "A multi-attribute group decisionmaking method based on weighted geometric aggregation operators of interval-valued trapezoidal fuzzy numbers", Applied Mathematical Modelling, 36(6), pp. 2498-2509 (2012).

46. Liu, P., Zhang, X., and Jin, F. "A multi-attribute group decision-making method based on intervalvalued trapezoidal fuzzy numbers hybrid harmonic averaging operators", Journal of Intelligent \& Fuzzy Systems, 23(5), pp. 159-168 (2012).

47. Du, W.S. "Minkowski-type distance measures for generalized orthopair fuzzy sets", International Journal of Intelligent Systems, 33(4), pp. 802-817 (2018).

48. Peng, X. and Dai, J. "Research on the assessment of classroom teaching quality with q-rung orthopair fuzzy information based on multiparametric similarity measure and combinative distance-based assessment", International Journal of Intelligent Systems, 34, pp. 1588-1630 (2019).

49. Joshi, B.P., Singh, A., Bhatt, P.K., et al. "Interval valued q-rung orthopair fuzzy sets and their properties", Journal of Intelligent \& Fuzzy Systems, 35(3), pp. 5225-5230 (2018).

50. Jaccard, P. "Distribution de la flore alpine dans le bassin des Dranses et dans quelques régions voisines", Bull. Soc. Vaudoise Sci. Nat., 37, pp. 241-272 (1901).

51. Dice, L.R. "Measures of the amount of ecologic association between species", Ecology, 26(3), pp. 297-302 (1945).

52. Salton, G. and McGill, M.J., Introduction to Modern Information Retrieval, mcGraw-hill (1983).

53. Xu, X., Zhang, L., and Wan, Q. "A variation coefficient similarity measure and its application in emergency group decision-making", Systems Engineering Procedia, 5, pp. 119-124 (2012).

54. Pramanik, S., Biswas, P., and Giri, B.C. "Hybrid vector similarity measures and their applications to multi-attribute decision making under neutrosophic environment", Neural Computing and Applications, 28(5), pp. 1163-1176 (2017).

55. Wei, G. and Wei, Y. "Similarity measures of Pythagorean fuzzy sets based on the cosine function and their applications", International Journal of Intelligent Systems, 33(3), pp. 634-652 (2018). 
56. Zhang, X. "A novel approach based on similarity measure for Pythagorean fuzzy multiple criteria group decision making", International Journal of Intelligent Systems, 31(6), pp. 593-611 (2016).

57. Liu, P. and You, X. "Linguistic neutrosophic partitioned Maclaurin symmetric mean operators based on clustering algorithm and their application to multicriteria group decision making", Artificial Intelligence Review, 53(3), pp. 2131-2170 (2020).

58. Liu, P. and Li, Y. "Multi-attribute decision making method based on generalized maclaurin symmetric mean aggregation operators for probabilistic linguistic information", Computers \& Industrial Engineering, 131, pp. 282-294 (2019).

59. Liu, P. and Zhang, X. "Multi-criteria decision making approach with linguistic D numbers based on choquet integral", Cognitive Computation, 11(4), pp. 560-575 (2019).

60. Liu, P., Gao, H., and Ma, J. "Novel green supplier selection method by combining quality function deployment with partitioned Bonferroni mean operator in interval type-2 fuzzy environment", Information Sciences, 490, pp. 292-316 (2019).

61. Liu, P., Zhu, B., Wang, P., et al. "An approach based on linguistic spherical fuzzy sets for public evaluation of shared bicycles in China", Engineering Applications of Artificial Intelligence, 87, p. 103295 (2020).

62. Liu, P. and Wang, Y. "Multiple attribute decision making based on q-rung orthopair fuzzy generalized Maclaurin symmetric mean operators", Information Sciences, 518, pp. 181-210 (2020).

63. Liu, P. and Liu, X. "A multi-attribute decision making method based on the third generation prospect theory and grey correlation degree", Scientia Iranica, 28(2), pp. 1001-1013 (2021).

64. Liu, P. and Liu, W. "Bonferroni harmonic mean operators based on two-dimensional uncertain linguistic information and their applications in land utilization ratio evaluation", Scientia Iranica, 26(2), pp. 975-995 (2019).

\section{Biographies}

Peide Liu received BS and MS degrees in Signal and Information Processing from Southeast University, Nanjing, China in 1988 and 1991, respectively, and the $\mathrm{PhD}$ degree in Information Management from Beijing Jiaotong University, Beijing, China in 2010. He is currently a Professor at the School of Management Science and Engineering, Shandong University of Finance and Economics, Shandong, China. He is an Associate Editor of the Information Sciences, International Journal of Fuzzy Sets, Journal of Intelligent and Fuzzy Systems, the editorial board of the journal Technological and Economic Development of Economy, and the member of the editorial board of other 12 journals. He has authored or coauthored more than 200 publications. His research interests include aggregation operators, fuzzy logic, fuzzy decision-making, and their applications.

Tahir Mahmood is an Assistant Professor of Mathematics at Department of Mathematics and Statistics, International Islamic University Islamabad, Pakistan. $\mathrm{He}$ received his $\mathrm{PhD}$ degree in Mathematics from Quaid-i-Azam University Islamabad, Pakistan in 2012 under the supervision of Professor Dr. Muhammad Shabir. His areas of interest are algebraic structures, fuzzy algebraic structures, and soft sets. He has more than 65 international publications to his credit and also produced 38 MS students.

Zeeshan Ali received the BS degree in Mathematics from Abdul Wali Khan University Mardan, Pakistan in 2016, and the MS degree in the same field from International Islamic University Islamabad, Pakistan in 2018. Currently, he is a Student of PhD in Mathematics in International Islamic University Islamabad, Pakistan. His research interests include aggregation operators, fuzzy logic, fuzzy decision-making, and their applications. He has published more than 10 articles. 